\title{
Input-specific control of interneuron numbers in nascent striatal networks
}

\author{
Varun Sreenivasan a,b, Eleni Serafeimidou-Pouliou a,b, David Exposito-Alonso a,b, \\ Kinga Bercsenyi ${ }^{a, b}$, Clémence Bernard ${ }^{a, b}$, Sung-Eun Bae ${ }^{a, b}$, Fazal Oozeer ${ }^{a, b}$, \\ Alicia Hanusz-Godoy ${ }^{\mathrm{a}, \mathrm{b}}$, Robert Edwards ${ }^{\mathrm{c}}$ and Oscar Marín a,b, ${ }^{*}$
}

aCentre for Developmental Neurobiology, Institute of Psychiatry, Psychology and Neuroscience, King's College London, London SE1 1UL, United Kingdom; ' ${ }^{M R C}$ Centre for Neurodevelopmental Disorders, King's College London, London SE1 1UL, United Kingdom; 'Department of Physiology and Department of Neurology, School of Medicine, University of California San Francisco, United States of America

*To whom correspondence should be addressed. Email: oscar.marin@kcl.ac.uk 
The assembly of functional neuronal circuits requires appropriate numbers of distinct classes of neurons, but the mechanisms through which their relative proportions are established remain poorly defined. Investigating the mouse striatum, here we found that the two most prominent subtypes of striatal interneurons, parvalbumin-expressing (PV+) GABAergic and cholinergic (ChAT+) interneurons, undergo extensive programmed cell death between the first and second postnatal weeks. Remarkably, the survival of PV+ and ChAT+ interneurons is regulated by distinct mechanisms mediated by their specific afferent connectivity. While long-range cortical inputs control PV+ interneuron survival, ChAT+ interneuron survival is regulated by local input from the medium spiny neurons. Our results identify input-specific circuit mechanisms that operate during the period of programmed cell death to establish the final number of interneurons in nascent striatal networks.

There are hundreds of different types of projection neurons and interneurons in the mammalian nervous system, but the various cellular components of each brain structure arise during development in very precise proportions. This is largely achieved by an evolutionarily conserved strategy based on the initial overproduction of cells and the subsequent elimination of a fraction of them through the process of programmed cell death (Oppenheim, 1991; Raff et al., 1993).

In the neocortex, the relative proportion of excitatory pyramidal neurons and inhibitory GABAergic interneurons is similar across cortical areas and even species (DeFelipe et al., 2002; Hendry et al., 1987). In mice, pyramidal cells and interneurons undergo extensive programmed cell death during early postnatal development to adjust their final ratio (Wong and Marín, 2019). Cortical interneurons appear to be intrinsically programmed to undergo cell death unless they sustain a certain level of activity during early postnatal development (Denaxa et al., 2018; Priya et al., 2018; Southwell et al., 2012; Wong et al., 2018). This mechanism guarantees that only interneurons that receive sufficient input from pyramidal cells are retained (Duan et al., 2020; Wong et al., 2018).

The striatum is another brain structure that contains both projection neurons and interneurons. However, it is unique in its almost complete lack of glutamatergic neurons. The principal neurons of the striatum, called medium spiny neurons (MSNs), are GABAergic and 
constitute $95 \%$ of all striatal neurons (Graveland and DiFiglia, 1985). Single-cell transcriptomic analyses have identified several populations of striatal interneurons in the mouse (Muñoz-Manchado et al., 2018), including two major types for which there is abundant functional, morphological, and electrophysiological information: (1) parvalbuminexpressing (PV+) GABAergic interneurons; and (2) choline acetyltransferase expressing $($ ChAT+) cholinergic interneurons (Kawaguchi, 1993). Like many cortical interneurons, striatal PV+ and ChAT+ interneurons derive from progenitor cells in the medial ganglionic eminence (MGE) and preoptic area (POA) of the embryonic telencephalon (Marín et al., 2000), but it is presently unknown whether these cells also undergo programmed cell death during early postnatal development. In addition, the absence of local excitatory neurons raises the question of how the appropriate ratio of projection neurons and interneurons is established in the striatum and whether the cellular mechanisms underlying this process are universal across the brain. Here we found that striatal interneurons undergo extensive programmed cell death, a process that is specifically regulated by their afferent connectivity during a critical window of early postnatal development. Our experiments reveal that activity-dependent, input-specific control of programmed cell death regulates interneuron numbers in the striatum.

\section{Results}

\section{Striatal interneurons undergo programmed cell death}

To investigate whether striatal interneurons undergo programmed cell death during postnatal development, we generated Nkx2-1-Cre; $R C L^{\text {tdTomato }}$ mice in which MGE/POA-derived interneurons are irreversibly labeled from early embryonic development and quantified the number of tdTomato-expressing interneurons in the striatum using stereological methods. Between postnatal days (P) 2 and P 21 (Fig. 1A) we observed a dramatic decrease in the total number of striatal interneurons. By the end of the third postnatal week, the total number of MGE/POA-derived interneurons in the striatum reduced to nearly half the number found around birth, with the most significant variation occurring between P5 and P10 (Fig. 1B and C).

We next sought to determine whether different populations of striatal MGE/POA-derived interneurons undergo programmed cell death at comparable rates. We hypothesized that like their cortical counterparts (Southwell et al., 2012; Wong et al., 2018), striatal interneurons might require the concerted action of the BCL2 family genes Bax and Bakl to undergo cell 
death. Consequently, removing Bax and Bakl in striatal interneurons would reveal how this process specifically affects distinct interneuron classes. To test this hypothesis, we generated Nkx2-1-Cre;Bakl $1^{-1 /} ; B_{a x}^{f l l f l}$ mutant mice and quantified the density of PV+ and ChAT+ striatal interneurons. Suppressing cell death in MGE/POA-derived cells led to a $30 \%$ increase in the density of PV+ interneurons (Fig. 1D and E) and a $40 \%$ increase in the density of ChAT+ interneurons (Fig. 1F and G), indicating that both PV+ and ChAT+ interneuron numbers are strongly modulated during early postnatal development.

\section{Cortical pyramidal neurons regulate striatal interneuron survival}

The survival of cortical GABAergic interneurons is regulated by the number and activity of local excitatory neurons (Wong et al., 2018). The striatum lacks an equivalent population of local excitatory neurons, but its main source of excitatory input originates from the neocortex along with prominent input from the thalamus (Johansson and Silberberg, 2020; Klug et al., 2018; Wall et al., 2013). To test if long-range cortical inputs regulate striatal interneuron survival, we first asked whether preventing cortical excitatory neurons from undergoing programmed cell death - thereby artificially increasing their number - would, in turn, change the survival of striatal interneurons. To this end, we quantified the density of PV+ and ChAT+ interneurons in $\mathrm{Nex}^{\mathrm{Crel+}} ; \mathrm{Bakl}^{-/-} ; \mathrm{Bax} \mathrm{fllfl}^{\mathrm{flf}}$ mice, in which the programmed cell death of cortical pyramidal cells is specifically abolished (Wong et al., 2018). Artificially increasing the number of pyramidal neurons during development led to a $20 \%$ increase in the density of $\mathrm{PV}+$ interneurons throughout the striatum (Fig. 2A and C). In contrast, we observed no changes in the density of ChAT+ interneurons (Fig. 2B and D).

The previous results revealed that the survival of striatal PV+ interneurons depends on the number of cortical pyramidal cells. $\mathrm{PV}+$ interneurons preferentially receive synaptic input from cortico-striatal projections (Johansson and Silberberg, 2020), which might explain the differences observed among interneuron subtypes. Therefore, we investigated whether increasing the activity of cortical pyramidal cells during the period of striatal interneuron cell death would affect their survival. To this end, we transiently increased the activity of pyramidal cells using the chemogenetic actuator hM3Dq (Roth, 2016). We expressed an adeno-associated virus (AAV) encoding Cre dependent hM3Dq in the frontal cortex of Nex ${ }^{\mathrm{Cre} /+}$ mice at P1 (Fig. 2E and F). We then injected pups with the ligand clozapine -N-oxide (CNO) or vehicle twice daily between P6 and P10 and examined the distribution of striatal interneurons at P21. Enhancing pyramidal cell activity during the period of striatal 
interneuron cell death led to a robust $\sim 24 \%$ increase in the density of PV+ interneurons (Fig. $2 \mathrm{G}$ and $\mathrm{I}$ ) and a more modest increase of $\sim 17 \%$ in the density of ChAT+ interneurons (Fig. $2 \mathrm{H}$ and $\mathrm{J})$.

To rule out the possibility that the observed increase in PV+ interneuron density is due to changes in PV levels and not in actual interneuron numbers, we quantified the density of striatal Nkx2-1+ cells that do not express ChAT. At P21, striatal Nkx2-1+/ChAT- cells comprise all PV+ interneurons and a very small percentage of somatostatin neurons (Marín et al., 2000). We found an increase in Nkx2-1+/ChAT- interneuron density in CNO-treated mice compared to controls (Fig. S1B-C) indicating that the increased density of striatal PV+ interneurons is likely due to increased survival and not to activity-dependent changes in PV levels.

\section{Paradoxical effects of cortical dysfunction on striatal interneuron survival}

We next investigated whether the modulation of striatal interneuron numbers by cortical pyramidal neurons is bi-directional. Based on our previous results, we hypothesized that decreasing the number or activity of pyramidal neurons, during the period of programmed cell death, would negatively impact PV+ and ChAT+ interneuron survival. To test this hypothesis, we first generated Rbp4-Cre;Stxbp $1^{f l f l}$ mice, in which syntaxin-binding protein 1 (also known as Munc18-1) is removed from Rbp4-expressing (Rbp4+) layer 5 pyramidal neurons in the neocortex. Deleting Stxbpl blocks neurotransmission, which is followed by rapid apoptosis of the cell (Verhage et al., 2000). By P6, we found that mutant mice contained significantly fewer Ctip2+ layer 5 pyramidal cells than control mice in primary somatosensory barrel cortex and primary motor cortex (Fig. S2A-B). We then assessed whether the loss of cortico-striatal projection neurons impacted the survival of striatal interneurons. Consistent with our hypothesis, we found that mutant mice contained $\sim 12 \%$ fewer striatal PV+ interneurons than controls at P21 (Fig. 3A and C). Unexpectedly, we also observed a $\sim 20 \%$ increase in the density of striatal ChAT+ interneurons in mutants compared to control mice (Fig. 3B and D).

Next, we tested whether decreasing the activity of pyramidal neurons has similar effects on striatal interneuron survival as reducing their number. We expressed Cre-dependent AAVs encoding the chemogenetic actuator hM4Di (Roth, 2016) throughout the neocortex of Rbp4Cre mice at P1 (Fig. 3E and F). We then injected pups with CNO or vehicle thrice daily between P6 and P10 and examined the distribution of striatal interneurons at P21. We found a 
significant decrease of $\sim 18 \%$ in the density of $\mathrm{PV}+$ interneurons in CNO-treated mice compared to controls (Fig. 3G and I). Interestingly, we again observed a significant increase of $\sim 14 \%$ in the density of striatal ChAT+ interneurons (Fig. $3 \mathrm{H}$ and J). Altogether, these experiments demonstrated that the survival of striatal PV+ interneurons can be bidirectionally modulated by the number and activity of cortical pyramidal neurons. These experiments also revealed paradoxical effects on ChAT+ interneurons since both bidirectional manipulations led to an increase in the survival of this population.

\section{PV+ interneuron survival requires glutamatergic neurotransmission}

In the neocortex, the activity of individual MGE interneurons correlates with their survival (Wong et al., 2018) and lowering their activity in a cell-autonomous manner reduces their ability to survive (Duan et al., 2020). Since the activity of any given neuron is largely derived from glutamatergic inputs, we first tested whether striatal interneuron survival depends on glutamatergic neurotransmission from the cortex. To delete the vesicular glutamate transporters Vglut1 and Vglut2, we crossed double heterozygous Vglut1 mutant and floxed Vglut2 mice, and injected Cre-expressing AAVs in the neocortex of the resulting control $\left(\right.$ Vglut $\left.^{+/+} ; V_{\text {glut }} 2^{+/+}\right)$and double mutant (Vglut1 $1^{-/} ; V_{\text {glut }}$ fllfl $\left.^{\prime}\right)$ animals at P1 (Fig. 4A and B). We found a very strong decrease of $\sim 70 \%$ in the density of PV+ interneurons in Cre-injected Vglut $^{-1-} ; V_{\text {Vglut }} 2^{f l l f l}$ mice compared to controls in the striatum (Fig. 4C and E) as well as in the cortex (Fig. S3A-B) at P21. In contrast, the loss of cortical glutamate led to a significant increase of $\sim 21 \%$ in the survival of striatal ChAT+ interneurons (Fig. 4D and F). These results revealed that glutamate neurotransmission in cortico-striatal neurons is crucial for the survival of striatal PV+ interneurons but not ChAT+ interneurons.

\section{ChAT+ interneuron survival depends on MSN activity}

Striatal ChAT+ interneurons receive relatively few direct inputs from pyramidal cells (Johansson and Silberberg, 2020; Lapper and Bolam, 1992), which suggests that the paradoxical effects on ChAT+ interneuron survival, caused by manipulating the cortex, might be indirect. We hypothesized that the survival of ChAT+ interneurons might be controlled by the activity of the MSNs since ChAT+ interneurons receive particularly strong inputs from these cells (Chuhma et al., 2011). We tested this hypothesis by injecting AAVs expressing hM3Dq in the striatum of Nkx2-1-Cre mice at P1 (Fig. 4G and H). To prevent the expression of hM3Dq in PV+ and ChAT+ interneurons, we flanked the expression construct with loxP sequences to excise it out in Cre expressing populations. We then injected pups with CNO or 
vehicle thrice daily between P6 and P10 and examined the distribution of striatal interneurons at P21. Activating MSNs during the period of striatal interneuron cell death did not impact the survival of PV+ interneurons (Fig. $4 \mathrm{I}$ and K). In contrast, we observed a prominent $\sim 38 \%$ decrease in the density of ChAT+ interneurons (Fig. 4J and L) suggesting that MSN activity, during this period, negatively impacts the survival of ChAT+ interneurons.

\section{Discussion}

Our study demonstrates that the neocortex can remotely influence the establishment of neural circuits in another region of the brain, the striatum. We found that the two most prominent types of striatal interneurons, PV+ GABAergic neurons and ChAT+ cholinergic neurons, undergo substantial programmed cell death during a short period of early postnatal development in the mouse. Their survival is under the control of specific afferent inputs during the cell death period. The final number of PV+ interneurons is established by longrange cortical inputs, while local inputs from medium spiny neurons regulate the final density of ChAT+ interneurons. Our results reveal circuit-based rules for establishing interneuron ratios and point to activity-dependent, input-specific mechanisms as the main determinant of the final number of interneurons in nascent striatal networks (Fig. S4).

\section{Long-range control of striatal PV+ interneuron survival}

Striatal PV+ interneurons mediate inhibition onto neighboring MSNs (Gittis et al., 2010; Koos and Tepper, 1999; Straub et al., 2016) and they are primarily driven by long-range excitatory inputs from the cortex and the thalamus (Johansson and Silberberg, 2020; Ramanathan et al., 2002; Rudkin and Sadikot, 1999). We found that modifying the number or activity of cortical pyramidal neurons bi-directionally regulates the survival of these interneurons. Given the key role these interneurons play in controlling MSN activity in response to cortical input (Gittis et al., 2010; Koos and Tepper, 1999; Mallet et al., 2005; Straub et al., 2016), it seems logical that pyramidal cells are directly involved in establishing their final numbers.

The neocortex controls the survival of striatal PV+ interneurons through glutamatergic neurotransmission. Preventing exocytotic glutamate release from cortical pyramidal cells led to the elimination of nearly $70 \%$ of striatal PV+ interneurons. Since these interneurons also receive excitatory inputs from the thalamus (Johansson and Silberberg, 2020; Rudkin and Sadikot, 1999; Sciamanna et al., 2015), it is conceivable that glutamate release from thalamic 
synapses might be sufficient to promote the survival of the remaining striatal PV+ interneurons in our experiments. In contrast, PV+ interneurons are not influenced by the local activity of their main target, the neighboring MSNs, presumably because they receive relatively few inputs from these cells (Chuhma et al., 2011; Taverna et al., 2007).

\section{Local control of striatal ChAT+ interneuron survival}

The final number of striatal ChAT+ interneurons is primarily regulated by the activity of the local MSNs. Increasing MSN activity negatively impacts ChAT+ interneuron survival. This result is consistent with the observation that $\mathrm{ChAT}+$ interneurons receive prominent inputs from these cells (Chuhma et al., 2011; Gonzales et al., 2013; Guo et al., 2015). The neocortex can influence the survival of striatal ChAT+ cells, although this effect is likely mediated by the indirect modulation of MSN activity. Several lines of evidence support this conclusion. First, pyramidal cells provide only weak and sparse direct excitatory inputs to ChAT+ interneurons (Johansson and Silberberg, 2020; Lapper and Bolam, 1992). Second, in the absence of excitatory synaptic drive, MSNs remain quiescent due to a very hyperpolarized resting membrane potential (Wilson and Kawaguchi, 1996). Consequently, in experiments where cortico-striatal inputs are reduced, it is expected that MSN activity would decrease, which would in turn increase the survival of ChAT+ cells. Third, since the number of striatal $\mathrm{PV}+$ interneurons scale up when the number or activity of pyramidal neurons are experimentally raised, increasing cortico-striatal inputs may paradoxically decrease the activity of MSNs, and indirectly increase the survival of ChAT+ interneurons.

It is tempting to speculate on the source of the signals promoting the survival of ChAT+ interneurons. One possibility is that glutamate release from thalamic synapses might contribute towards the survival of these interneurons, as these cells receive more substantial inputs from the thalamus compared to the relatively weak cortical innervation (Johansson and Silberberg, 2020; Lapper and Bolam, 1992). Alternatively, it has been shown that ChAT+ interneurons are tonically active (Aosaki et al., 1995; Wilson et al., 1990). This spontaneous activity is generated by endogenous ionic conductances (Bennett et al., 2000; Wilson, 2005), independently of excitatory synapses (Bennett et al., 2000; Bennett and Wilson, 1999). It is therefore conceivable that the endogenous firing of striatal ChAT+ interneurons might promote the survival of these cells during the period of programmed cell death. 


\section{Outlook}

Striatal interneuron dysfunction is associated with neurodevelopmental disorders that affect movement, cognition, and behavior. Alterations in striatal interneuron numbers have been described in patients with Tourette syndrome and schizophrenia (Holt et al., 2005; Kalanithi et al., 2005; Kataoka et al., 2010). Equally, experimental manipulation of the activity and number of these cells leads to motor stereotypies and other functional deficits in mice (Aliane et al., 2011; Crittenden et al., 2017; Rapanelli et al., 2017). In the striatum, the regulation of $\mathrm{PV}+$ interneuron numbers is important for establishing balanced ratios of excitation and inhibition. Furthermore, this balance appears to be crucial for the survival of ChAT+ interneurons. Elucidating whether similar mechanisms operate in humans may shed new light on the neurobiology of neurodevelopmental disorders with alterations in the number of interneurons. 


\section{ACKNOWLEDGMENTS}

We thank I. Andrews for excellent technical assistance; S. Anderson (University of Pennsylvania, USA), K. Nave (Max Planck Institute for Experimental Medicine, Germany) and M. Verhage (Vrije Universiteit Amsterdam, The Netherlands) for Nkx2-1-Cre, Nex Cre/t and Stxbp $1^{f l f l}$ mice, respectively; N. Dehorter and B. Rico for critical reading of the manuscript, and members of the Marín and Rico laboratories for stimulating discussions and ideas. Supported by a grant from the European Research Council (ERC-2017-AdG 787355) to O.M.

Data Availability. All materials, code, and data will be shared upon reasonable request.

Author Contributions: V.S., K.B. and O.M. conceived the study. K.B. and V.S. performed initial experiments establishing the programmed cell death of striatal interneurons. V.S. performed all experiments described in this manuscript. D.E-A., F.O., C.B., and A.H-G. contributed to cloning and validation of the Cre-out shRNA constructs. E.S-P. and S-E.B. contributed to data collection. R.E. contributed Vglut1 and Vglut2 mice. V.S. and O.M. wrote the paper with input from all authors.

Competing Interest Statement: The authors declare no competing interests. 


\section{References}

Aliane, V., Perez, S., Bohren, Y., Deniau, J.M., and Kemel, M.L. (2011). Key role of striatal cholinergic interneurons in processes leading to arrest of motor stereotypies. Brain 134, 110118.

Aosaki, T., Kimura, M., and Graybiel, A.M. (1995). Temporal and spatial characteristics of tonically active neurons of the primate's striatum. J. Neurophysiol. 73, 1234-1252.

Bennett, B.D., Callaway, J.C., and Wilson, C.J. (2000). Intrinsic membrane properties underlying spontaneous tonic firing in neostriatal cholinergic interneurons. J. Neurosci. 20, 8493-8503.

Bennett, B.D., and Wilson, C.J. (1999). Spontaneous activity of neostriatal cholinergic interneurons in vitro. J. Neurosci. 19, 5586-5596.

Chuhma, N., Tanaka, K.F., Hen, R., and Rayport, S. (2011). Functional connectome of the striatal medium spiny neuron. J. Neurosci. 31, 1183-1192.

Crittenden, J.R., Lacey, C.J., Weng, F.J., Garrison, C.E., Gibson, D.J., Lin, Y., and Graybiel, A.M. (2017). Striatal Cholinergic Interneurons Modulate Spike-Timing in Striosomes and Matrix by an Amphetamine-Sensitive Mechanism. Front. Neuroanat. 11, 20.

DeFelipe, J., Alonso-Nanclares, L., and Arellano, J.I. (2002). Microstructure of the neocortex: comparative aspects. J. Neurocytol. 31, 299-316.

Denaxa, M., Neves, G., Rabinowitz, A., Kemlo, S., Liodis, P., Burrone, J., and Pachnis, V. (2018). Modulation of apoptosis controls inhibitory interneuron number in the cortex. Cell Reports 22, 1710-1721.

Duan, Z.R.S., Che, A., Chu, P., Modol, L., Bollmann, Y., Babij, R., Fetcho, R.N., Otsuka, T., Fuccillo, M.V., Liston, C., et al. (2020). GABAergic restriction of network dynamics regulates interneuron survival in the developing cortex. Neuron 105, 75-92 e75.

Gittis, A.H., Nelson, A.B., Thwin, M.T., Palop, J.J., and Kreitzer, A.C. (2010). Distinct roles of GABAergic interneurons in the regulation of striatal output pathways. J. Neurosci. 30, 2223-2234. 
Gonzales, K.K., Pare, J.F., Wichmann, T., and Smith, Y. (2013). GABAergic inputs from direct and indirect striatal projection neurons onto cholinergic interneurons in the primate putamen. J. Comp. Neurol. 521, 2502-2522.

Graveland, G.A., and DiFiglia, M. (1985). The frequency and distribution of medium-sized neurons with indented nuclei in the primate and rodent neostriatum. Brain Res. 327, 307-311.

Guo, Q., Wang, D., He, X., Feng, Q., Lin, R., Xu, F., Fu, L., and Luo, M. (2015). Wholebrain mapping of inputs to projection neurons and cholinergic interneurons in the dorsal striatum. PLoS One 10, e0123381.

Hendry, S.H., Schwark, H.D., Jones, E.G., and Yan, J. (1987). Numbers and proportions of GABA-immunoreactive neurons in different areas of monkey cerebral cortex. J. Neurosci. 7, 1503-1519.

Holt, D.J., Bachus, S.E., Hyde, T.M., Wittie, M., Herman, M.M., Vangel, M., Saper, C.B., and Kleinman, J.E. (2005). Reduced density of cholinergic interneurons in the ventral striatum in schizophrenia: an in situ hybridization study. Biol. Psychiatry 58, 408-416.

Johansson, Y., and Silberberg, G. (2020). The functional organization of cortical and thalamic inputs onto five types of striatal neurons is determined by source and target cell identities. Cell Reports 30, 1178-1194 e1173.

Kalanithi, P.S., Zheng, W., Kataoka, Y., DiFiglia, M., Grantz, H., Saper, C.B., Schwartz, M.L., Leckman, J.F., and Vaccarino, F.M. (2005). Altered parvalbumin-positive neuron distribution in basal ganglia of individuals with Tourette syndrome. Proc. Natl. Acad. Sci. USA $102,13307-13312$.

Kataoka, Y., Kalanithi, P.S., Grantz, H., Schwartz, M.L., Saper, C., Leckman, J.F., and Vaccarino, F.M. (2010). Decreased number of parvalbumin and cholinergic interneurons in the striatum of individuals with Tourette syndrome. J. Comp. Neurol. 518, 277-291.

Kawaguchi, Y. (1993). Physiological, morphological, and histochemical characterization of three classes of interneurons in rat neostriatum. J. Neurosci. 13, 4908-4923.

Klug, J.R., Engelhardt, M.D., Cadman, C.N., Li, H., Smith, J.B., Ayala, S., Williams, E.W., Hoffman, H., and Jin, X. (2018). Differential inputs to striatal cholinergic and parvalbumin interneurons imply functional distinctions. Elife 7.

Koos, T., and Tepper, J.M. (1999). Inhibitory control of neostriatal projection neurons by GABAergic interneurons. Nat. Neurosci. 2, 467-472. 
Lapper, S.R., and Bolam, J.P. (1992). Input from the frontal cortex and the parafascicular nucleus to cholinergic interneurons in the dorsal striatum of the rat. Neuroscience 51,533 545.

Mallet, N., Le Moine, C., Charpier, S., and Gonon, F. (2005). Feedforward inhibition of projection neurons by fast-spiking GABA interneurons in the rat striatum in vivo. J. Neurosci. $25,3857-3869$.

Marín, O., Anderson, S.A., and Rubenstein, J.L.R. (2000). Origin and molecular specification of striatal interneurons. J. Neurosci. 20, 6063-6076.

Muñoz-Manchado, A.B., Bengtsson Gonzales, C., Zeisel, A., Munguba, H., Bekkouche, B., Skene, N.G., Lonnerberg, P., Ryge, J., Harris, K.D., Linnarsson, S., and Hjerling-Leffler, J. (2018). Diversity of interneurons in the dorsal striatum revealed by single-cell RNA sequencing and patchSeq. Cell Reports 24, 2179-2190 e2177.

Oppenheim, R.W. (1991). Cell death during development of the nervous system. Annu. Rev. Neurosci. 14, 453-501.

Priya, R., Paredes, M.F., Karayannis, T., Yusuf, N., Liu, X., Jaglin, X., Graef, I., AlvarezBuylla, A., and Fishell, G. (2018). Activity regulates cell death within cortical interneurons through a Calcineurin-dependent mechanism. Cell Reports 22, 1695-1709.

Raff, M.C., Barres, B.A., Burne, J.F., Coles, H.S., Ishizaki, Y., and Jacobson, M.D. (1993). Programmed cell death and the control of cell survival: lessons from the nervous system. Science 262, 695-700.

Ramanathan, S., Hanley, J.J., Deniau, J.M., and Bolam, J.P. (2002). Synaptic convergence of motor and somatosensory cortical afferents onto GABAergic interneurons in the rat striatum. J. Neurosci. 22, 8158-8169.

Rapanelli, M., Frick, L.R., and Pittenger, C. (2017). The role of interneurons in autism and tourette syndrome. Trends Neurosci 40,397-407.

Roth, B.L. (2016). DREADDs for Neuroscientists. Neuron 89, 683-694.

Rudkin, T.M., and Sadikot, A.F. (1999). Thalamic input to parvalbumin-immunoreactive GABAergic interneurons: organization in normal striatum and effect of neonatal decortication. Neuroscience $88,1165-1175$. 
Sciamanna, G., Ponterio, G., Mandolesi, G., Bonsi, P., and Pisani, A. (2015). Optogenetic stimulation reveals distinct modulatory properties of thalamostriatal vs corticostriatal glutamatergic inputs to fast-spiking interneurons. Sci Rep 5, 16742.

Southwell, D.G., Paredes, M.F., Galvao, R.P., Jones, D.L., Froemke, R.C., Sebe, J.Y., AlfaroCervello, C., Tang, Y., Garcia-Verdugo, J.M., Rubenstein, J.L., et al. (2012). Intrinsically determined cell death of developing cortical interneurons. Nature 491, 109-113.

Straub, C., Saulnier, J.L., Begue, A., Feng, D.D., Huang, K.W., and Sabatini, B.L. (2016). Principles of synaptic organization of GABAergic interneurons in the striatum. Neuron 92 , 84-92.

Taverna, S., Canciani, B., and Pennartz, C.M. (2007). Membrane properties and synaptic connectivity of fast-spiking interneurons in rat ventral striatum. Brain Res. 1152, 49-56.

Verhage, M., Maia, A.S., Plomp, J.J., Brussaard, A.B., Heeroma, J.H., Vermeer, H., Toonen, R.F., Hammer, R.E., van den Berg, T.K., Missler, M., et al. (2000). Synaptic assembly of the brain in the absence of neurotransmitter secretion. Science $287,864-869$.

Wall, N.R., De La Parra, M., Callaway, E.M., and Kreitzer, A.C. (2013). Differential innervation of direct- and indirect-pathway striatal projection neurons. Neuron 79, 347-360.

Wilson, C.J. (2005). The mechanism of intrinsic amplification of hyperpolarizations and spontaneous bursting in striatal cholinergic interneurons. Neuron 45, 575-585.

Wilson, C.J., Chang, H.T., and Kitai, S.T. (1990). Firing patterns and synaptic potentials of identified giant aspiny interneurons in the rat neostriatum. J. Neurosci. 10, 508-519.

Wilson, C.J., and Kawaguchi, Y. (1996). The origins of two-state spontaneous membrane potential fluctuations of neostriatal spiny neurons. J. Neurosci. 16, 2397-2410.

Wong, F.K., Bercsenyi, K., Sreenivasan, V., Portales, A., Fernandez-Otero, M., and Marin, O. (2018). Pyramidal cell regulation of interneuron survival sculpts cortical networks. Nature $557,668-673$.

Wong, F.K., and Marín, O. (2019). Developmental cell death in the cerebral cortex. Annu. Rev. Cell. Dev. Biol. 35, 523-542. 

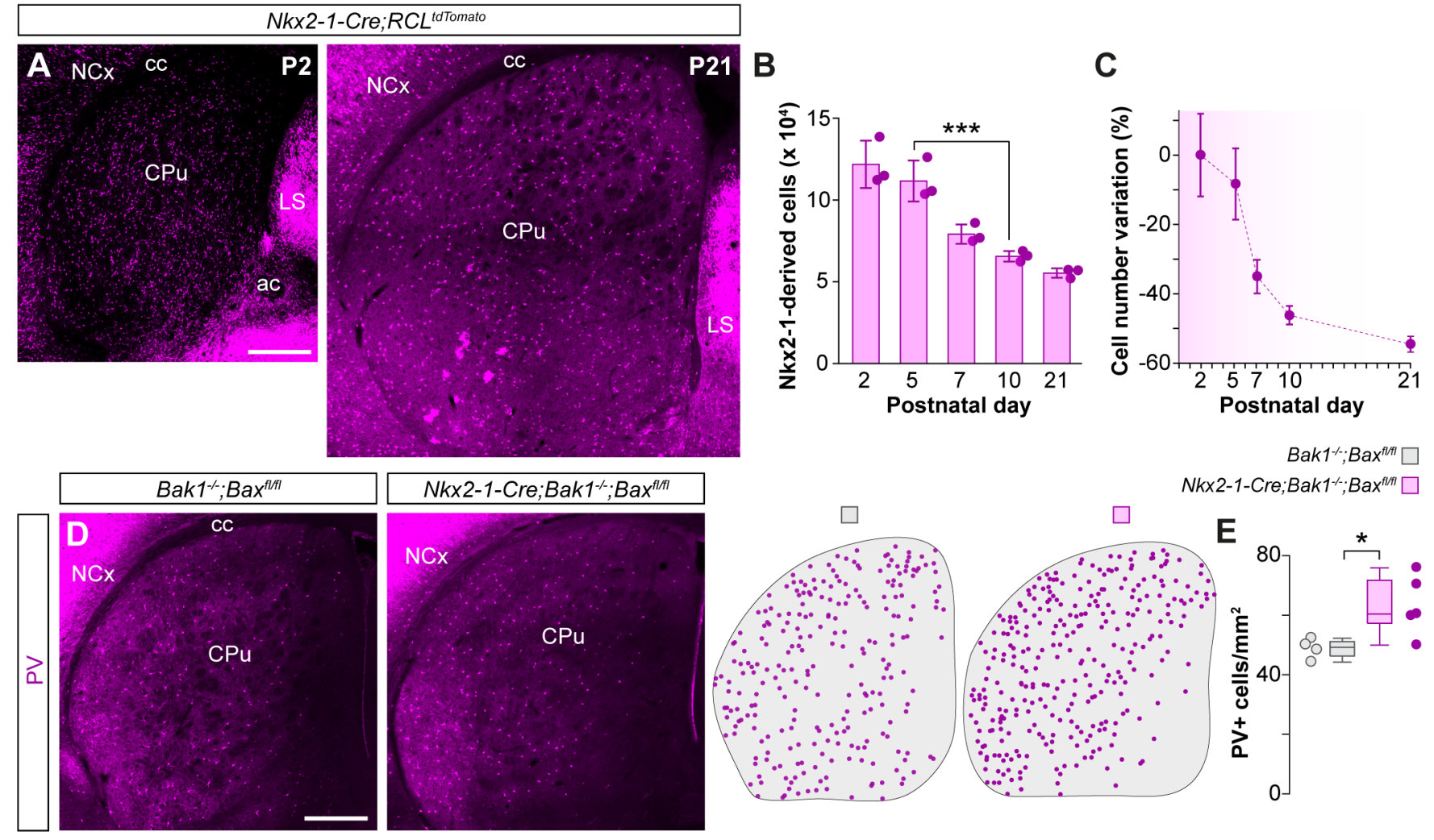

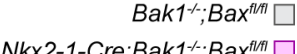
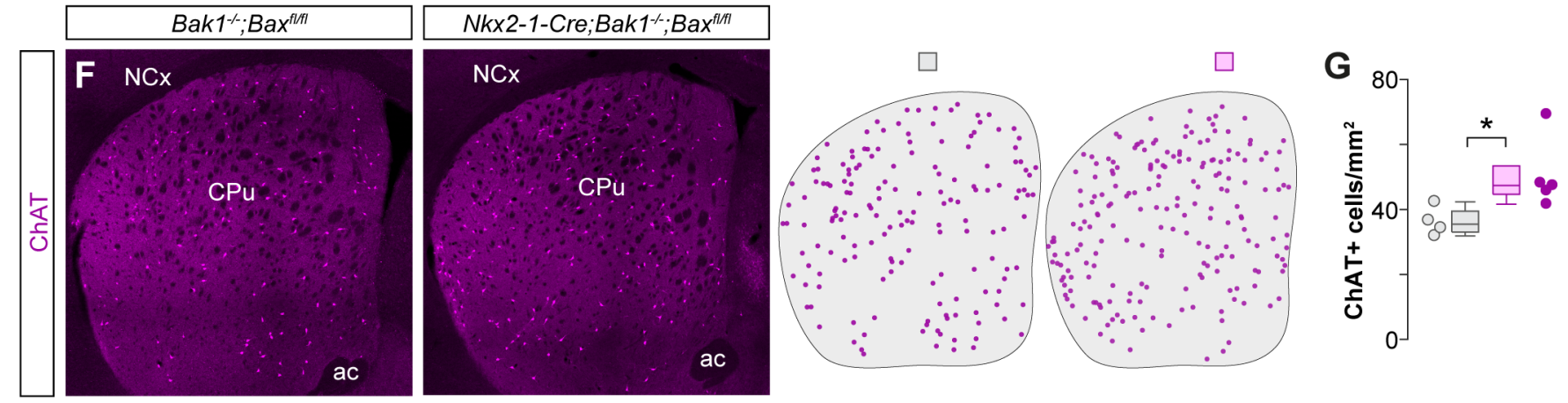

Figure 1 


\section{Fig. 1. Striatal interneurons undergo programmed cell death during early postnatal}

development. (A) Coronal sections through the striatum of $N k x 2-1-C r e ; R C L^{\text {TdTomato }}$ mice at P2 and P21. (B) Stereological estimates of the total number of tdTomato+ neurons in the striatum of Nkx2-1-Cre;RCL TdTomato mice at different postnatal stages ( $n=3$ mice per postnatal stage); one-way ANOVA, *** $p<0.001$; P2 vs. P5: Tukey-Kramer HSD test, $p>0.05$; P5 vs. P10: Tukey-Kramer HSD test, ${ }^{* * *} p<0.001$. (C) Percentage variation in the number of tdTomato+ cells at different postnatal stages relative to the average number of tdTomato+ cells at P2

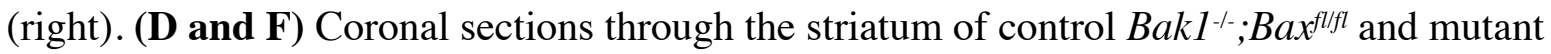
Nkx2-1-Cre;Bakl $1^{-/} ;$Bax $^{f l f l}$ mice immunostained for PV (D) and ChAT (F). The schematic dot maps indicate the locations of striatal interneurons in each case. (E and G) Quantification of

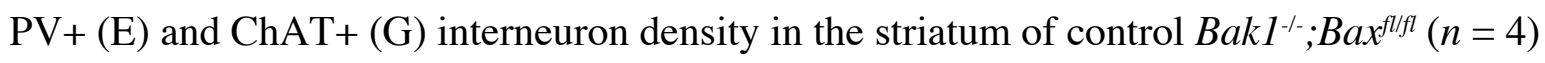
and mutant Nkx2-1-Cre;Bakl ${ }^{-1-} ; B a x^{f l l f l}(n=5)$ mice. PV+ interneurons: two-tailed unpaired Student's $t$-test, ${ }^{*} p<0.05$. ChAT+ interneurons: Wilcoxon's rank-sum test, ${ }^{*} p<0.05$. Data in panel $\mathrm{B}$ are shown as bar plots and data in panels $\mathrm{E}$ and $\mathrm{G}$ as box plots. Adjacent data points indicate the stereological estimate in each mouse (B) or the average cell density in each animal (E and G). Error bars indicate standard deviation. Scale bars, $500 \mu \mathrm{m}$. 

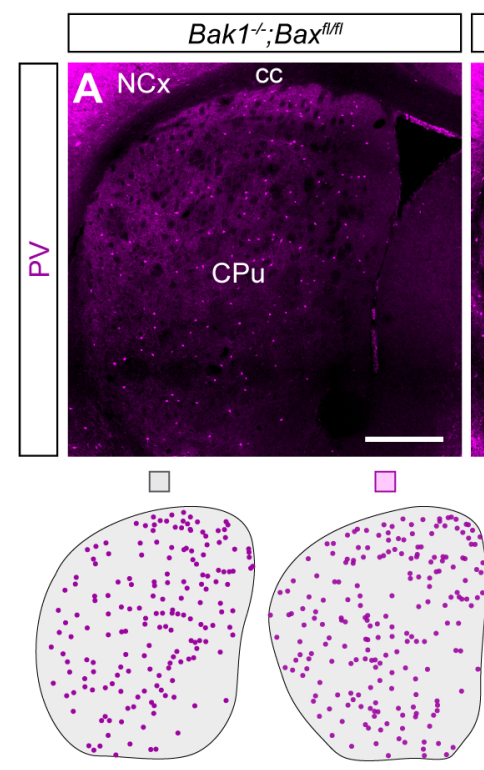

E

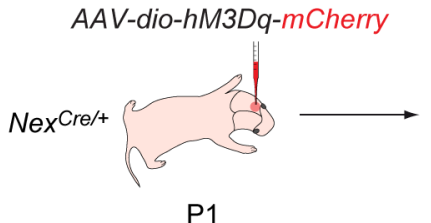

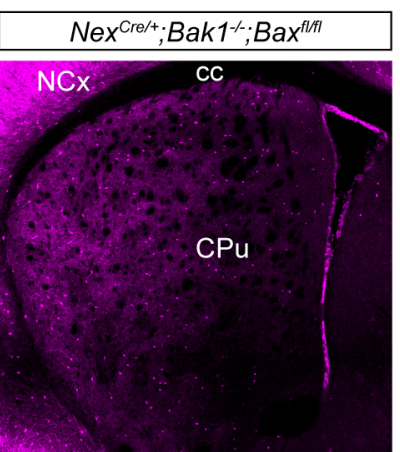

C

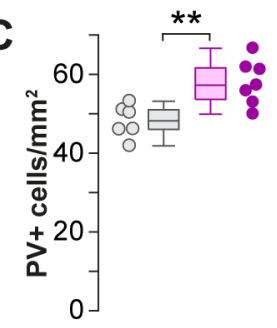

CNO or vehicle

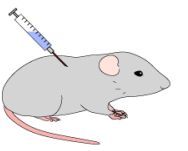

P6 to P10
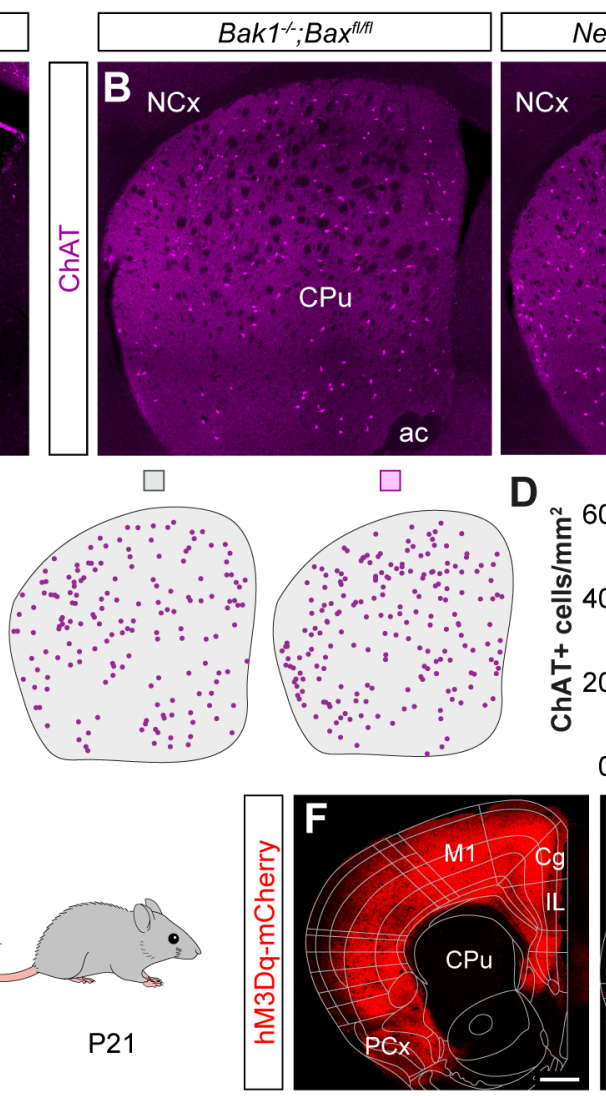

P21
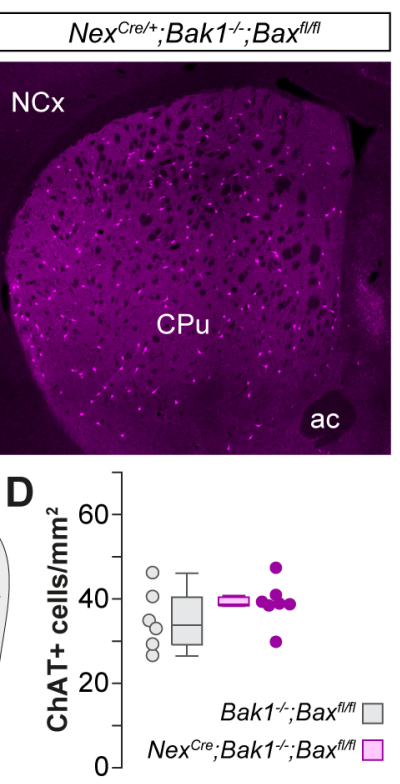
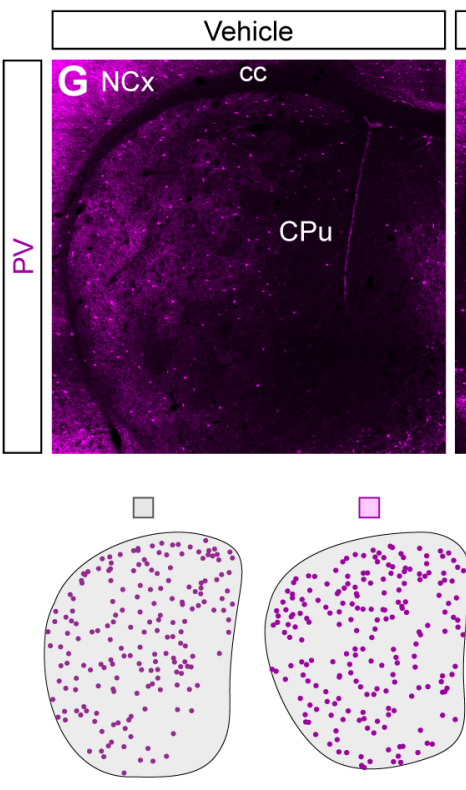

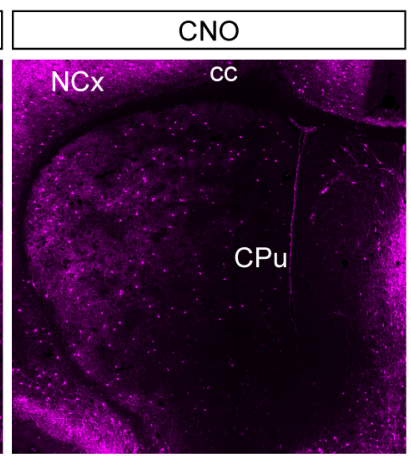

I

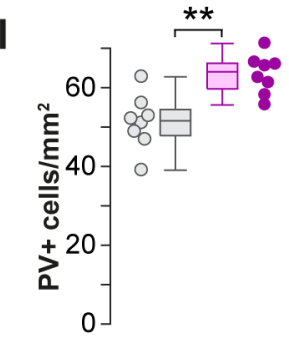

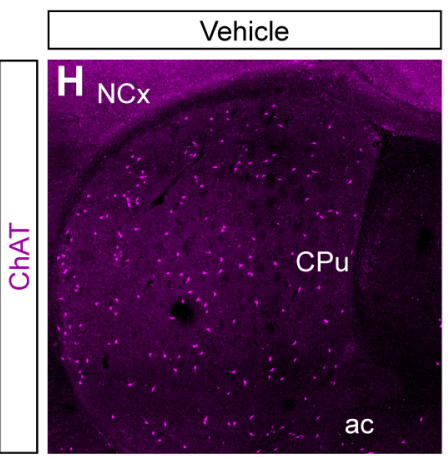

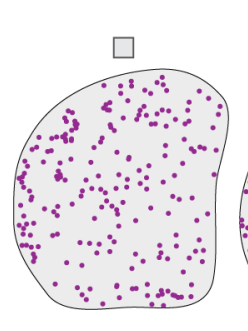

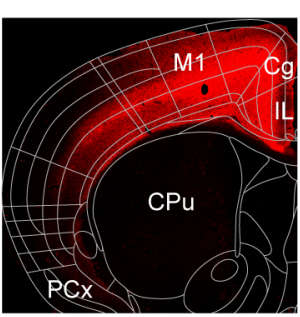

CNO
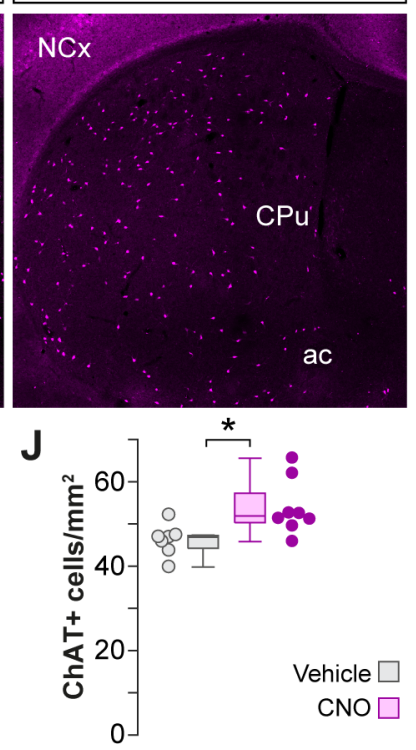

Figure 2 
Fig. 2. Cortical gain of function rescues striatal PV+ and ChAT+ interneurons. (A and B) Coronal sections through the striatum of control $B a k 1^{-1-} ; B a x^{f l l f l}$ mice and mutant $\mathrm{Nex}^{\mathrm{Crel+}} ; \mathrm{Bakl}^{-/-} ; \mathrm{Bax}^{\mathrm{fllfl}}$ mice immunostained for PV (A) and ChAT (B). The schematic dot maps indicate the locations of striatal interneurons in each case. (C and D) Quantification of $\mathrm{PV}+(\mathrm{C})$ and ChAT+ (D) interneuron density in the striatum of control $\operatorname{Bakl}^{-/-} ; \operatorname{Bax}^{f l f l}(n=6)$ and mutant $\mathrm{Nex}^{\mathrm{Crel+}} ; \mathrm{Bakl}^{-/ /} ; \mathrm{Bax}^{f l / f l}(n=7)$ mice. PV+ interneurons: two-tailed unpaired Student's $t$-test, ${ }^{* *} p<0.01$. ChAT+ interneurons: two-tailed unpaired Student's $t$-test, $p=$ 0.27. (E) Schematic of experimental design. (F) Coronal sections through the brain at $\sim 1.5$ $\mathrm{mm}$ (left) and $\sim 0.5 \mathrm{~mm}$ (right) anterior to Bregma showing the extent of the viral infection. The images have been overlaid with the corresponding coronal maps. (G and $\mathbf{H})$ Coronal sections through the striatum of vehicle and $\mathrm{CNO}$-treated $\mathrm{Nex}^{\mathrm{Cre} /+}$ mice immunostained for PV (G) and ChAT (H). The schematic dot maps indicate the locations of striatal interneurons in each case. (I and J) Quantification of PV+ (I) and ChAT+ (J) interneuron density in the striatum of vehicle ( $n=8$ for PV and 7 for ChAT) and CNO-treated $(n=8) \mathrm{Nex}^{\mathrm{Cre} /+}$ mice. PV+ interneurons: two-tailed unpaired Student's $t$-test, ${ }^{* *} p<0.01$. ChAT+ interneurons: twotailed unpaired Student's $t$-test, ${ }^{*} p<0.05$. Data in panels C, D, I and J are shown as box plots and the adjacent data points indicate the average cell density in each animal. Scale bars, 500 $\mu \mathrm{m}$. 

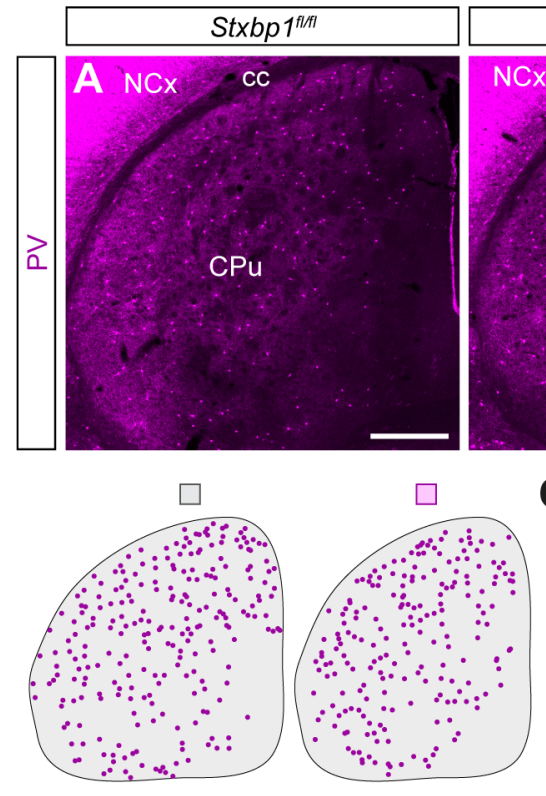

E

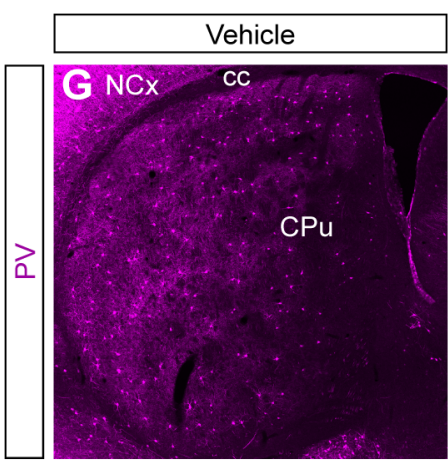

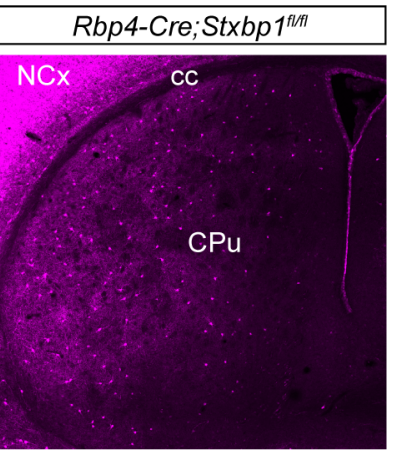

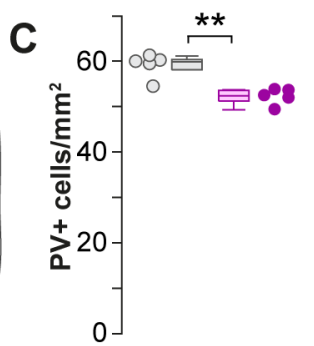

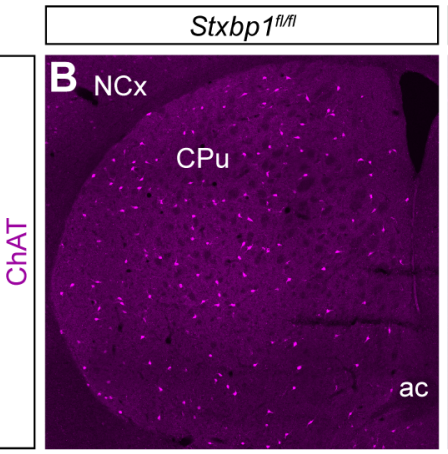

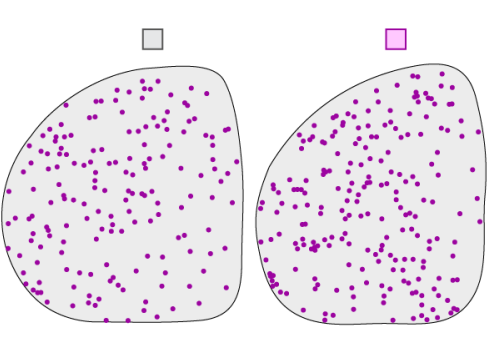

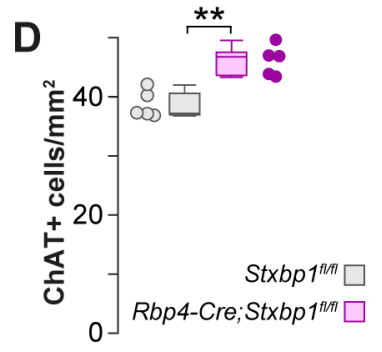

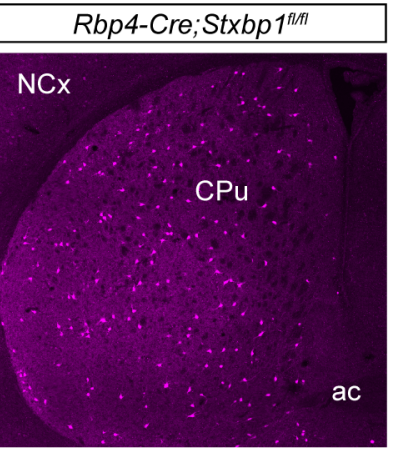

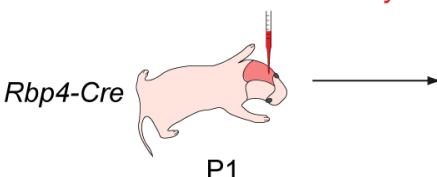

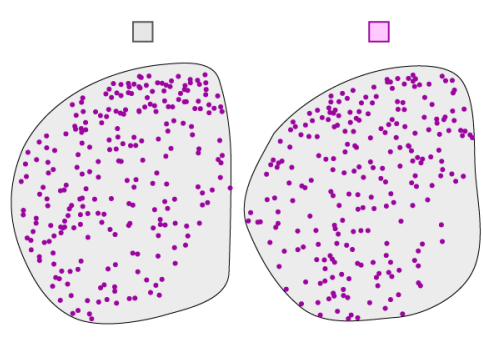

\section{AAV-dio-hM4Di-mCherry}

$\mathrm{P} 1$
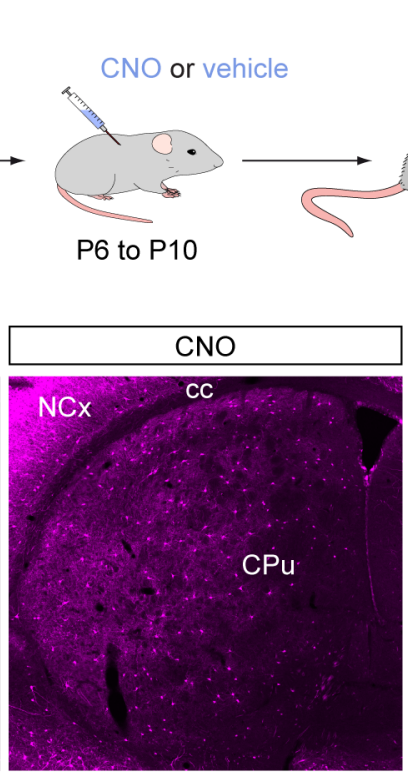

I
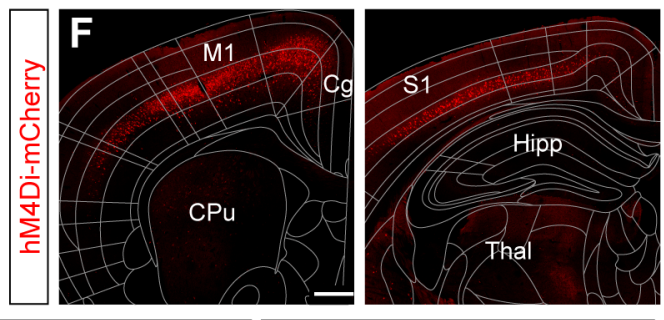

P21
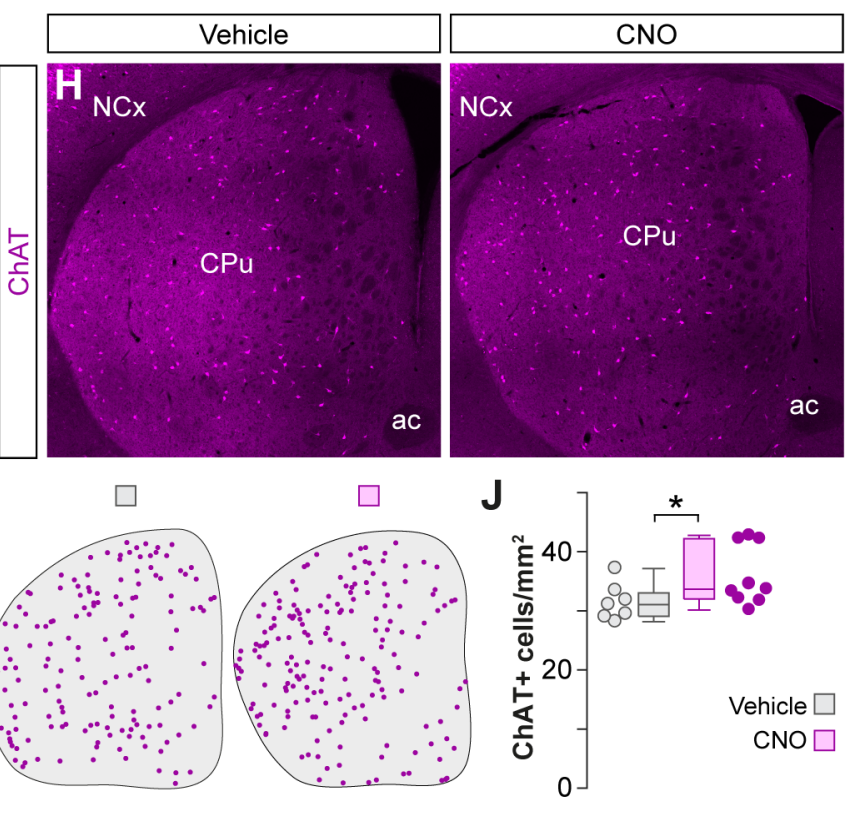

\section{Figure 3}




\section{Fig. 3. Cortical dysfunction differentially impacts the survival of striatal PV+ and}

ChAT+ interneurons. (A and B) Coronal sections through the striatum of control Stxbp $1^{f l f l}$ mice and mutant Rbp4-Cre;Stxbp $1^{f l f l}$ mice immunostained for PV (A) and ChAT (B). The schematic dot maps indicate the locations of striatal interneurons in each case. (C and D) Quantification of PV+ (C) and ChAT+ (D) interneuron density in the striatum of control Stxbp $1^{f l f l}(n=5)$ and mutant Rbp4-Cre;Stxbp $1^{f l / f l}(n=5)$ mice. PV+ interneurons: Wilcoxon's rank-sum test, ${ }^{* *} p<0.01$. ChAT+ interneurons: two-tailed unpaired Student's $t$-test, ${ }^{*} p<<$ 0.01. (E) Schematic of experimental design. (F) Coronal sections through the brain at $\sim 1 \mathrm{~mm}$ anterior (left) and $\sim 2 \mathrm{~mm}$ posterior (right) to Bregma showing the extent of the viral infection. The images have been overlaid with the corresponding coronal maps. (G and H) Coronal sections through the striatum of vehicle- and CNO-treated Rbp4-Cre mice immunostained for PV $(\mathrm{G})$ and ChAT $(\mathrm{H})$. The schematic dot maps indicate the locations of striatal interneurons in each case. (I and J) Quantification of PV+ (I) and ChAT+ (J) interneuron density in the striatum of vehicle $(n=7)$ and CNO-treated $(n=9)$ Rbp4-Cre mice. PV+ interneurons: twotailed unpaired Student's $t$-test, ${ }^{*} p<0.05$. ChAT+ interneurons: Wilcoxon's rank-sum test, ${ }^{*} p$ $=0.05$. Data in panels $\mathrm{C}, \mathrm{D}, \mathrm{I}$ and $\mathrm{J}$ are shown as box plots and the adjacent data points indicate the average cell density in each animal. Scale bars, $500 \mu \mathrm{m}$. 
A

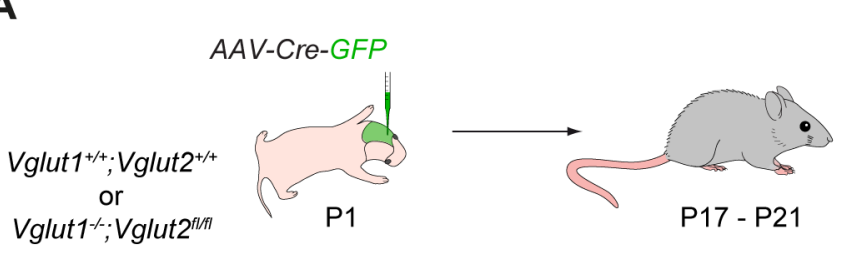

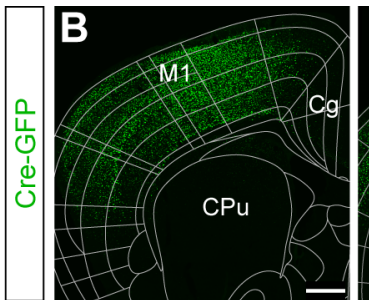
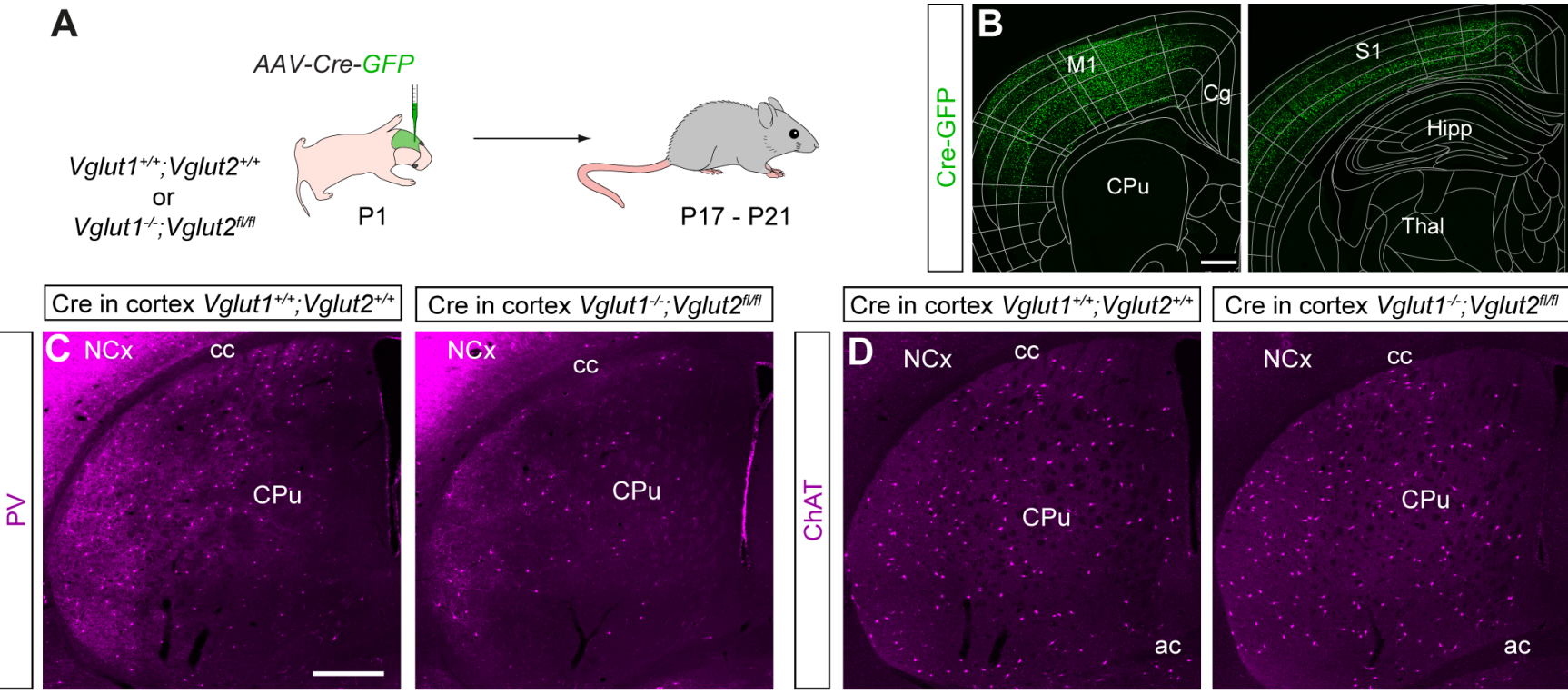

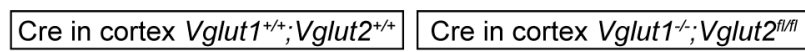
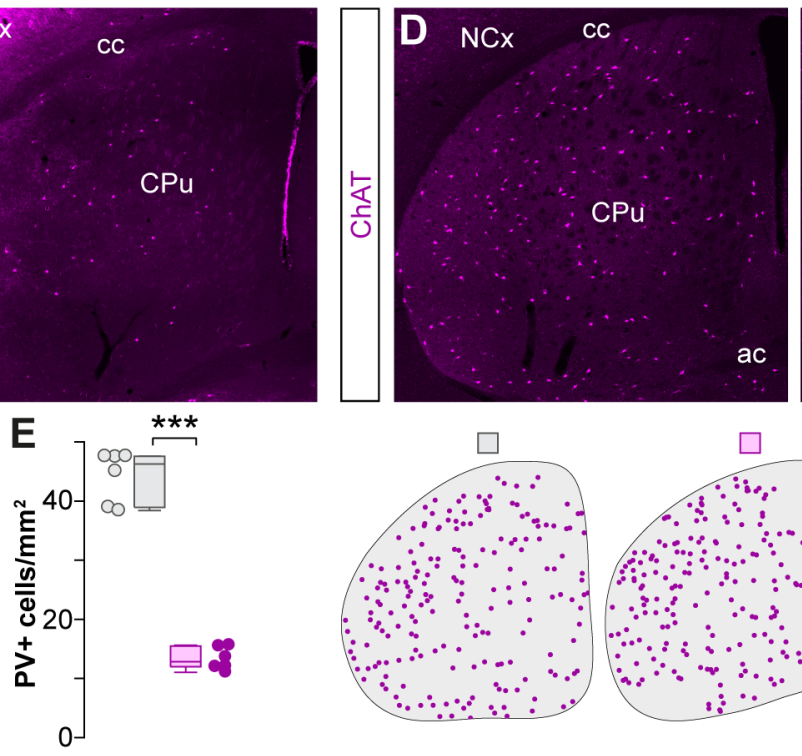

NCX

cc

$\mathrm{CPu}$
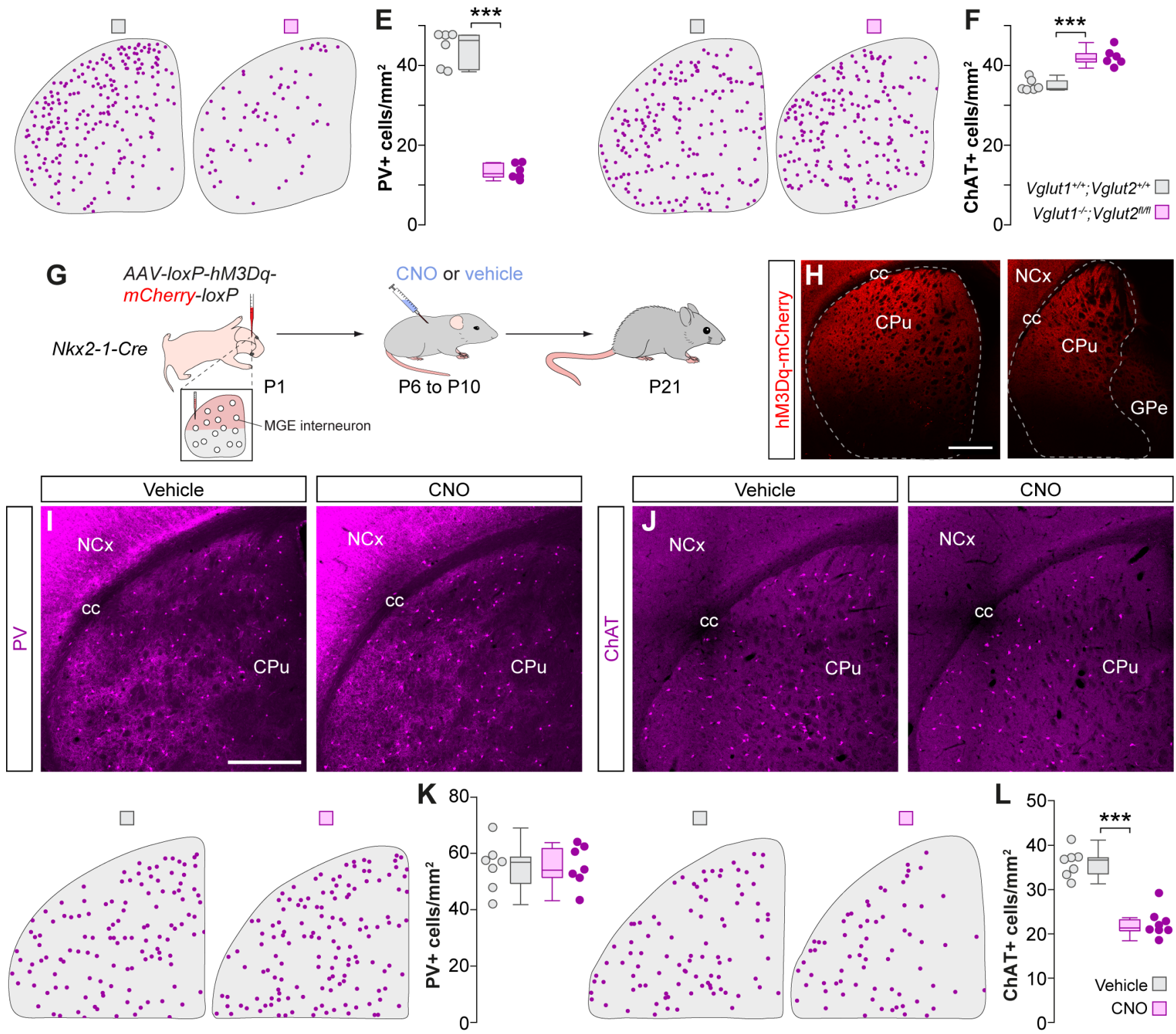
Fig. 4. Input specific mechanisms for striatal interneuron survival. (A) Schematic of experimental design. (B) Coronal sections through the brain at $\sim 1 \mathrm{~mm}$ anterior (left) and $\sim 2$ $\mathrm{mm}$ posterior (right) to Bregma showing the extent of the viral infection. The images have been overlaid with the corresponding coronal maps. (C and D) Coronal sections through the

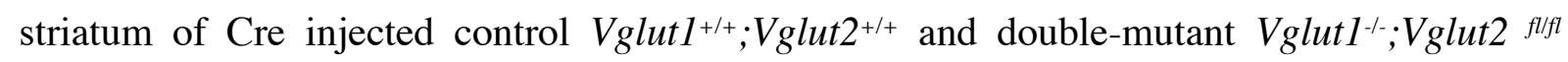
mice immunostained for PV (C) and ChAT (D). The schematic dot maps indicate the locations of striatal interneurons in each case. (E and F) Quantification of PV+ (E) and $\mathrm{ChAT}+(\mathrm{F})$ interneuron density in the striatum of Cre injected control Vglut1 ${ }^{+/+} ; \operatorname{Vglut} 2^{+/+}(n=$ 6) and double-mutant Vglut $^{-1 /} ;$ Vglut2 $^{\text {flfl } l}(n=6)$ mice. PV+ interneurons: two-tailed unpaired Student's $t$-test, ${ }^{* * *} p<0.001$. ChAT+ interneurons: two-tailed unpaired Student's $t$-test, $* * * p$ $<0.001$. (G) Schematic of experimental design. (H) Coronal sections through the striatum showing the extent of the viral infection. Dashed lines indicate the boundary of the striatum. (I and J) Coronal sections through the dorsal striatum of vehicle- and CNO-treated Nkx2-1Cre mice immunostained for PV (I) and ChAT (J). The schematic dot maps indicate the locations of striatal interneurons in each case. ( $\mathbf{K}$ and $\mathbf{L})$ Quantification of PV+ $(\mathrm{K})$ and $\mathrm{ChAT}+(\mathrm{L})$ interneuron density in the dorsal striatum of vehicle $(n=7)$ and CNO-treated $(n=$ 7 for PV and 8 for ChAT) Nkx2-1-Cre mice. PV+ interneurons: two-tailed unpaired Student's $t$-test, $p=0.9$. ChAT+ interneurons: two-tailed unpaired Student's $t$-test, ${ }^{* * *} p<0.001$. Data in panels $\mathrm{E}, \mathrm{F}, \mathrm{K}$ and $\mathrm{L}$ are shown as box plots and the adjacent data points indicate the average cell density in each animal. Scale bars, $500 \mu \mathrm{m}$. 


\title{
Input-specific control of interneuron numbers in nascent striatal networks
}

\author{
Varun Sreenivasan a,b, Eleni Serafeimidou-Pouliou a,b, David Exposito-Alonso a,b, \\ Kinga Bercsenyi ${ }^{a, b}$, Clémence Bernard ${ }^{a, b}$, Sung-Eun Bae ${ }^{a, b}$, Fazal Oozeer ${ }^{a, b}$, \\ Alicia Hanusz-Godoy ${ }^{a, b}$, Robert Edwardsc and Oscar Marín a,b, ${ }^{*}$
}

aCentre for Developmental Neurobiology, Institute of Psychiatry, Psychology and Neuroscience, King's College London, London SE1 1UL, United Kingdom; 'MRC Centre for Neurodevelopmental Disorders, King's College London, London SE1 1UL, United Kingdom; 'Department of Physiology and Department of Neurology, School of Medicine, University of California San Francisco, United States of America 


\section{A}

B

AAV-dio-hM3Dq-mCherry
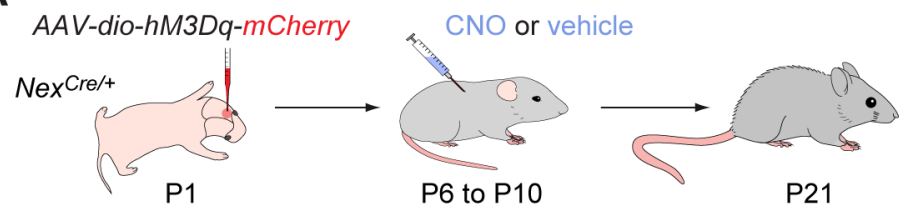

$\mathrm{P} 6$ to $\mathrm{P} 10$

P21

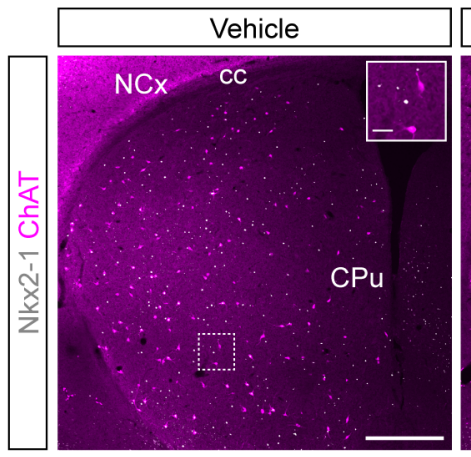

$\square$

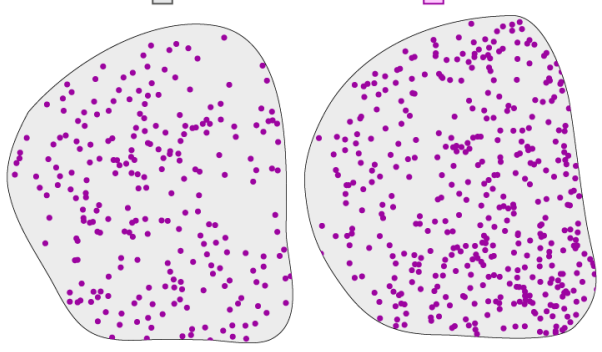

C
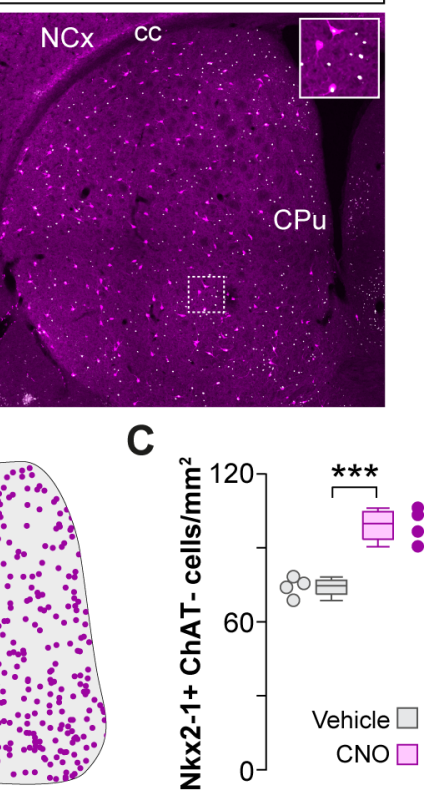

\section{Supplementary Figure 1}


Fig. S1. Increase in PV+ interneurons is due to rescue from cell death and not due to activity-related changes in PV+ expression. (A) Schematic of experimental design. (B) Coronal sections through the striatum of vehicle and $\mathrm{CNO}$ treated $\mathrm{Nex} \mathrm{Crel+}^{2}$ mice immunostained for Nkx2-1 and ChAT. The schematic dot maps indicate the locations of Nkx2-1+/ChATinterneurons in each case. (C) Quantification of Nkx2-1+/ChAT- interneuron (putative PV+ cell) density in the striatum of vehicle $(n=4)$ and CNO treated $(n=4) \mathrm{Nex}{ }^{\mathrm{Cre} /+}$ mice. Two-tailed unpaired Student's $t$-test, ${ }^{* * *} p<0.001$. Data are shown as boxplots and adjacent data points indicate the average cell density in each animal. Scale bars, $500 \mu \mathrm{m}$ and $50 \mu \mathrm{m}$ (inset). 
bioRxiv preprint doi: https://doi.org/10.1101/2021.12.01.470575; this version posted December 2, 2021. The copyright holder for this preprint (which was not certified by peer review) is the author/funder. All rights reserved. No reuse allowed without permission.

A P6 Primary somatosensory cortex (S1) P6 Primary motor cortex (M1)

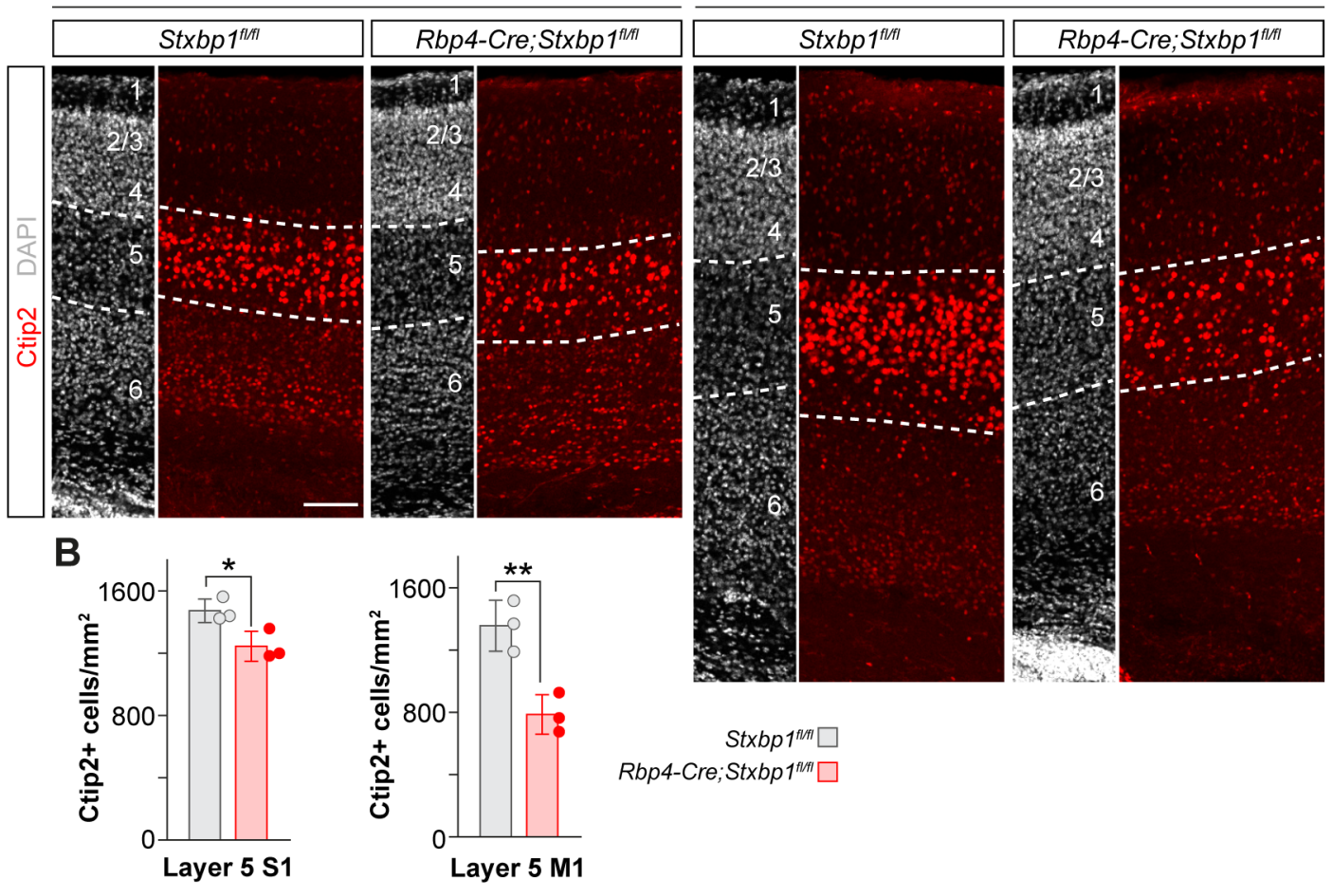

Supplementary Figure 2 
Fig. S2. Deleting Stxbp1 causes the death of layer 5 neurons by postnatal day 6. (A) Coronal sections through primary somatosensory cortex (S1) and primary motor cortex (M1) of control Stxbp $1^{f l f l}$ and Rbp4-Cre;Stxbp $1^{f l f l}$ mice immunostained for Ctip2 at P6. (B) Quantification of layer 5 Ctip2+ cell density in S1 and M1 of control Stxbp $1^{f l f l}(n=3)$ and mutant Rbp4Cre;Stxbp $1^{f l f l}(n=3)$ mice. S1: two-tailed unpaired Student's $t$-test, $* p<0.05$. M1: two-tailed unpaired Student's $t$-test, ${ }^{* *} p<0.01$. Data are shown as bar plots and adjacent data points indicate the average cell density in each animal. Error bars indicate standard deviation. Scale bar, $100 \mu \mathrm{m}$. 
A

Primary somatosensory cortex (S1)

B

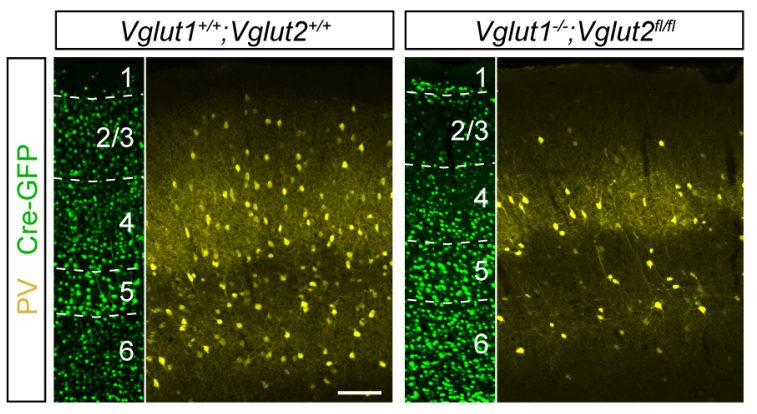

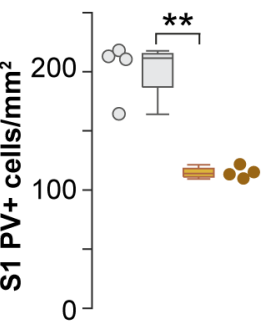

Primary motor cortex (M1)

Vglut1 ${ }^{++*} ; V_{\text {glut }} 2^{++4} \square$

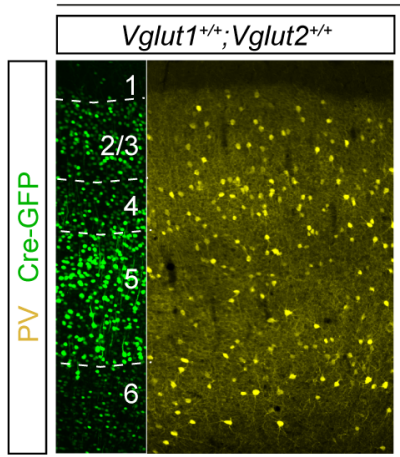

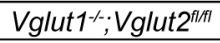

Vglut1-‘Vglut2

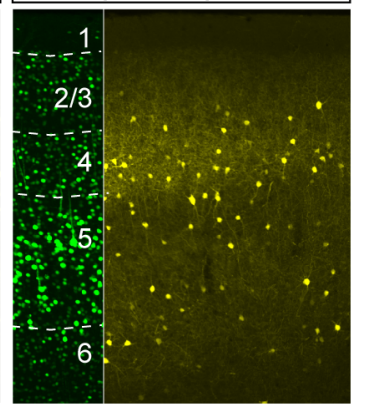

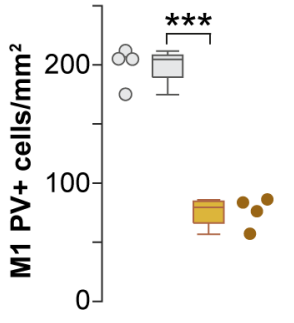

Supplementary Figure 3 
Fig. S3. Abolishing cortical glutamatergic transmission impacts the survival of cortical PV+ interneurons. (A) Coronal sections through primary somatosensory cortex (S1) and primary motor cortex (M1) of Cre injected control Vglut $^{+/+} ; \operatorname{Vglut}^{+/+}$and double-mutant Vglut1 $^{-/-}$;Vglut $2^{f l f l}$ mice. (B) Quantification of PV+ interneuron density in S1 and M1 of control $\operatorname{Vglut1}^{+/+} ; \operatorname{Vglut}^{+/+}(n=4)$ and double-mutant Vglut1 $1^{-/} ; V_{\text {Vlut }} 2^{f l f l}(n=4)$ mice. S1: two-tailed unpaired Student's $t$-test, ${ }^{* *} p<0.01$; M1: two-tailed unpaired Student's $t$-test, ${ }^{* * *} p<0.001$. Data are shown as boxplots and adjacent data points indicate the average cell density in each animal. Scale bar, $100 \mu \mathrm{m}$. 
bioRxiv preprint doi: https://doi.org/10.1101/2021.12.01.470575; this version posted December 2, 2021. The copyright holder for this preprint (which was not certified by peer review) is the author/funder. All rights reserved. No reuse allowed without permission.
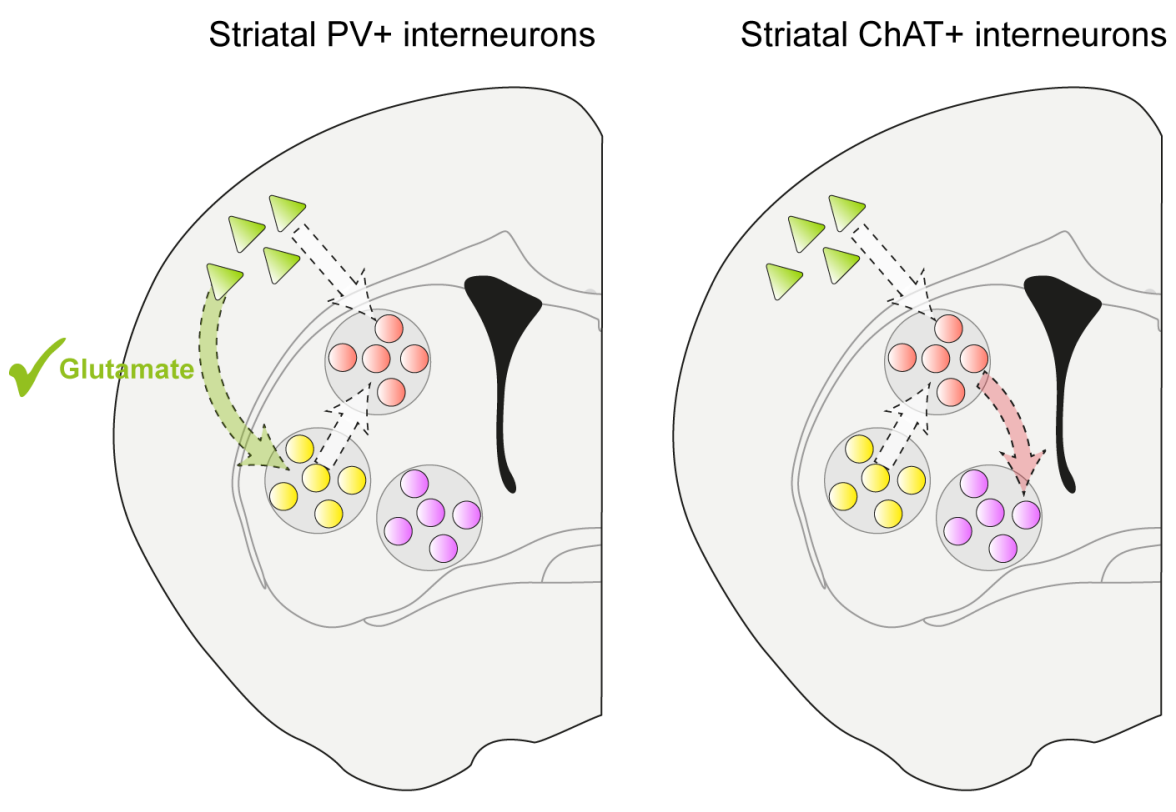

Pyramidal cell

Medium spiny neuron $\bigcirc$

PV interneuron $\bigcirc$

ChAT interneuron $\bigcirc$

Synaptic input is:

Synaptic + survival input

Synaptic + death input

Supplementary Figure 4 
Fig. S4. Circuit mechanisms regulating programmed cell death of striatal interneurons.

Striatal PV+ interneurons receive glutamatergic input from cortical pyramidal neurons that rescue them from programmed cell death and help to establish appropriate levels of inhibition in the striatum (left). The activity of striatal medium spiny neurons (MSNs) is tightly regulated by a combination of excitatory glutamatergic inputs from the cortex and inhibitory GABAergic inputs from PV+ interneurons. An imbalance leading to hyperactivity in MSNs kills ChAT+ interneurons (right). 


\section{Materials and Methods}

Mice. The mouse lines Nex $\mathrm{Crel}^{\mathrm{C}}\left(\right.$ Neurod6 $\left.{ }^{\text {tml(cre)Kan }}\right)$ (Goebbels et al., 2006), Nkx2-1-Cre

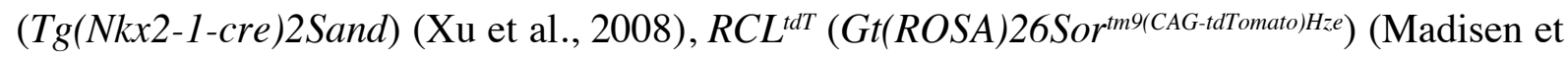
al., 2010), Bak $k^{-/}, B a x^{f l f l}$ (Bakl $1^{\text {tmlThsn }}$ and Bax ${ }^{\text {tm2Sjk})}$ (Takeuchi et al., 2005), Rbp4-Cre (Tg(Rbp4-

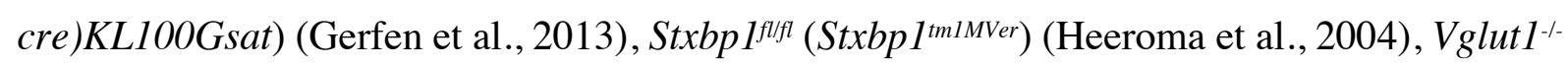
;Vglut2 $2^{\text {fllfl }}$ (Slc17a7tmlEdw and Slc17a6 $6^{\text {tml.IThna }}$ ) (Fremeau et al., 2004; Hnasko et al., 2010) were used in this study. Animals were housed in groups of up to five littermates and maintained under standard, temperature-controlled, laboratory conditions. Mice were kept on a 12:12 light/dark cycle and received water and food ad libitum. All animal procedures were approved by the ethical committee (King's College London) and conducted following European regulations, and Home Office personal and project licenses under the UK Animals (Scientific Procedures) 1986 Act.

Generation of DNA constructs. The Addgene plasmid $p A A V h S y n-h M 3 D(G q)$-mCherry (Addgene 50474) (Roth, 2016) was used as a starting point to generate pAAV hSyn-lox$h M 3 D(G q)$-mCherry-lox. Addgene_50474 was first digested with Sal1 and EcoR1 to generate a 4545 bp backbone for further cloning. The following primers were used to PCR amplify a fragment of 2597 bp containing $h M 3 D(G q)$-mCherry flanked by loxP sites in the same directional orientation:

$\mathrm{Fw}$ primer:

5 CTAGAGTCGACATAACTTCGTATAGCATACATTATACGAAGTTATGCCACCATGA CCTTGCAC-3'. Rv primer: 5' TATCGAATTCATAACTTCGTATAATGTATGCTATACGAAGTTATTTACTTGTACAG CTCGTCC-3'. The fragments were purified using QIAquick gel extraction kit (QIAGEN, Cat\# 28704) The PCR amplified fragment was digested with Sal1 and EcoR1 and ligated with the backbone to give the final construct.

AAV production. AAVs were produced using polyethylenimine (PEI) transfection of HEK293FT cells as described before (Favuzzi et al., 2019). In brief, DNA and PEI were mixed in the ratio of 1:4 in uncomplemented DMEM and left at room temp for 25 mins to form the DNA-PEI complex. The transfection solution was added to each plate and incubated for $72 \mathrm{~h}$ at $37^{\circ} \mathrm{C}$ in $5 \% \mathrm{CO} 2$. The transfected cells were then scraped off the plates and pelleted. The cell pellet was lysed in buffer containing $50 \mathrm{mN}$ Tris-Cl, $150 \mathrm{mM} \mathrm{NaCl}$ and $2 \mathrm{mM} \mathrm{MgCl} 2$ and $0.5 \%$ sodium deoxycholate and incubated with $100 \mathrm{U} / \mathrm{ml}$ Benzonase (Sigma-Aldrich, Cat\# E1014 25KU) for $1 \mathrm{~h}$ to dissociate particles from membranes. Any remaining contaminants and 
empty or incomplete viral particles were removed with a discontinuous iodixanol (OptiPrepTM) (Sigma-Aldrich, Cat\# D1556) gradient ultracentrifugation using four layers of different iodixanol concentrations of 15, 25, 40 and 58\% (Zolotukhin et al., 1999). The viral suspension was loaded on the iodixanol gradient in Quick-seal polyallomer tubes (BeckmanCoulter, Cat\# 342414) and spun in a VTi-50 rotor at 50,000 rpm for 75 mins at $12{ }^{\circ} \mathrm{C}$ in an Optima L-100 XP Beckman Coulter ultracentrifuge. The recovered virus fraction was purified by first passing through a 100-kDa molecular weight cut off (MWCO) centrifugal filter (Sartorius Vivaspin, Cat\# VS2041) and then through an Amicon Ultra 2ml Centrifugal filters (Merck Millipore, Cat\# UFC210024). Storage buffer (350 mM NaCl and 5\% Sorbitol in PBS) was added to the purified virus and stored in $5 \mu 1$ aliquots at $-80^{\circ} \mathrm{C}$. AAV titer was determined by quantitative polymerase chain reaction (qPCR) using primers for the WPRE sequence that is common to the construct. The following primers were used: WPRE Forward primer: 5'GGCACTGACAATTCCGTGGT-3'. WPRE Reverse primer: 5'CGCTGGATTGAGGGCCGAAG-3'. The extracted viral DNA and a serial dilution of the transfer plasmid DNA containing the WPRE sequence were transferred to a 96 well plate and measured using a LightCycler ${ }^{\circledR} 96$ instrument (Roche Life Science). AAVs produced had a titer of $2.10 \times 10^{13}$ viral genomes $/ \mathrm{ml}$.

Stereology. The total number of striatal MGE interneurons was estimated using the optical dissector method (West and Gundersen, 1990)

$$
N=\frac{\sum Q^{-} \cdot t}{h \cdot a s f . s s f}
$$

where $\Sigma \mathrm{Q}^{-}$is the total number of cells counted, $t$ the mean section thickness, $h$ the height of the optical dissector $(18 \mu \mathrm{m})$ adjusting for the guard zones $(2 \mu \mathrm{m})$ above and below the dissector, asf stands for the area sampling fraction and ssf stands for the section sampling fraction (sampling frequency). An ApoTome (Zeiss) microscope equipped with a motorized stage and color camera was connected to a computer with the Stereo Investigator software (MBF Biosciences). The boundaries of the striatum were first defined with a $2.5 \mathrm{x}$ objective (Zeiss) and the entire area was split into 4 equal quadrants.

Intracranial injections. P1 mice were anesthetized with 3\% isofluorane and mounted on a stereotaxic frame. Isoflurane concentration was maintained between 1 and $2 \%$ throughout the procedure. Injections of $h S y n-D I O-h M 3 D(G q)$-mCherry were targeted to the frontal cortex, at $\sim 0.5 \mathrm{~mm}$ anterior and $1 \mathrm{~mm}$ lateral to bregma, of the left hemisphere. $600 \mathrm{nl}$ of virus were 
injected at a depth of $400 \mu \mathrm{m}$ in the left hemisphere at a speed of $3 \mathrm{nl} \mathrm{sec}^{-1}$. Injections of $h S y n-$ DIO-hM4D(Gi)-mCherry were targeted to 6 sites in the left hemisphere to infect layer 5 neurons in the entire cortex. At each site, $200 \mathrm{nl}$ of virus were injected at a depth of $600 \mu \mathrm{m}$ at a speed of $3 \mathrm{nl} \mathrm{sec}^{-1}$. Injections of $h S y n-G F P-C r e$ were targeted to 6 sites in the left hemisphere to infect neurons in the entire cortex. At each site, $200 \mathrm{nl}$ of virus were injected at a depth of $400 \mu \mathrm{m}$ at a speed of $3 \mathrm{nl} \mathrm{sec}{ }^{-1}$. Injections of $h$ Syn-lox- $h M 3 D(G q)$-mCherry-lox were targeted to a single site in the left striatum. Along the anteroposterior axis, the site was located halfway between bregma and the blood vessel lying immediately posterior to the olfactory bulb and at $\sim 1.3$ to $1.5 \mathrm{~mm}$ lateral to the midline. $600 \mathrm{nl}$ of virus were injected at a depth of $\sim 1.4$ to $1.6 \mathrm{~mm}$ at a speed of $3 \mathrm{nl} \mathrm{sec}^{-1}$ to infect neurons in the dorsal striatum.

CNO injections. Injected animals were randomly assigned to the "Vehicle" or "CNO" groups. Clozapine-N-oxide (Tocris Bioscience, Cat\# 4936) was dissolved in $0.9 \%$ saline and $0.5 \%$ DMSO (Sigma-Aldrich, Cat\# D8418) to give a final concentration of $0.5 \mathrm{mg} \mathrm{ml}^{-1}$. The vehicle consisted of $0.5 \%$ DMSO in saline without the active ligand. In experiments where the activating DREADD hM3D $(\mathrm{Gq})$ was expressed in cortex, $\mathrm{CNO}$ or Vehicle was administered subcutaneously twice a day from P6 until P10 at a dose of $1 \mathrm{mg} \mathrm{kg}^{-1}$. This period was chosen since P6 and P10 correspond to the start and end of striatal interneuron death, respectively. In experiments where the $\mathrm{hM} 3 \mathrm{D}(\mathrm{Gq})$ was expressed in the striatum, CNO or Vehicle was administered subcutaneously thrice a day from P6 until P10 at a dose of $5 \mathrm{mg} \mathrm{kg}^{-1}$. In experiments where the inactivating DREADD $\mathrm{hM} 4 \mathrm{D}(\mathrm{Gi})$ was expressed in cortex, $\mathrm{CNO}$ or Vehicle was administered subcutaneously thrice a day from P6 until P10 at a dose of $5 \mathrm{mg} \mathrm{kg}-$ 1 .

Histology and immunohistochemistry. Mice were deeply anesthetized with pentobarbital sodium (Euthatal, Merial Animal Health Ltd) by intraperitoneal injection, and were transcardially perfused with $0.9 \% \mathrm{NaCl}$ solution (Sigma-Aldrich, Cat\# S76530) followed by 4\% paraformaldehyde (PFA) (Sigma-Aldrich, Cat\# 441244) dissolved in phosphatase-buffered saline (PBS). Brains were extracted and post-fixed for $2 \mathrm{~h}$ at $4^{\circ} \mathrm{C}$. The brains were immersed overnight in 30\% sucrose (Sigma-Aldrich, Cat\# S0389) and coronal sections were cut frozen on a sliding microtome (Leica SM2010 R) to a thickness of $40 \mu \mathrm{m}$. Every 8th section was used for antibody staining. The sampling was therefore every $280 \mu \mathrm{m}$. Free-floating brain slices were permeabilized with $0.25 \%$ Triton X-100 (Sigma-Aldrich, Cat\# T8787) in PBS for 30 mins, and blocked for $1 \mathrm{~h}$ in a blocking buffer containing $0.25 \%$ Triton $\mathrm{X}-100,10 \%$ normal donkey serum (Sigma-Aldrich, Cat\# S30), and 5\% bovine serum albumin (BSA) (Sigma-Aldrich, Cat\# 
A8806). Primary antibodies were dissolved in fresh blocking buffer and the sections were incubated overnight at $4^{\circ} \mathrm{C}$ in the primary antibody solution. The following day, the tissue was repeatedly rinsed. Secondary antibodies were dissolved in fresh blocking buffer and the sections were incubated in the secondary antibody solution for $2 \mathrm{~h}$ at room temperature. The sections were counterstained with $5 \mu \mathrm{M}$ 4',6-diamidine-2'-phenylindole dihydrochloride (DAPI) (Sigma-Aldrich, Cat\# D9542) in PBS, rinsed repeatedly and mounted on a glass slide with Mowiol and DABCO. The following primary antibodies were used: Rabbit anti-parvalbumin (1:1000, Swant, Cat\# PV-27), goat anti-ChAT (1:500, Millipore, Cat\# AB144P), rabbit antiTTF1 (1:500, Abcam, Cat\# ab76013), rat anti-Ctip2 (1:1000, Abcam, Cat\# ab18465). The following secondary antibodies were used: Donkey-anti-rabbit-Alexa 555 (1:400, Molecular Probes, Cat\# 31572), donkey anti-rabbit-Alexa 488 (1:400, Thermo Fisher Scientific, Cat\# 21206), donkey anti-rabbit-Alexa 647 (1:400, Thermo Fisher Scientific, Cat\# 31573), donkeyanti-goat Alexa 488 (1:400, Molecular Probes, Cat\# 11055), donkey-anti-goat Alexa 555 (1:400, Invitrogen, Cat\# 21432), donkey-anti-goat Alexa 647 (1:400, Thermo Fisher Scientific, Cat\# 21447), donkey-anti-rat Cy3 (1:400, Jackson ImmunoResearch Europe Ltd., Cat\# 712165-150).

Image acquisition and image analysis. Tile scan images of the striatum were acquired at 1,024x1,024-pixel resolution and 8-bit depth on an inverted Leica TCS-SP8 confocal microscope. 7 to 8 sections covering the striatum along the rostrocaudal axis were imaged. Samples from the same experimental litter were imaged and analyzed in parallel, using similar laser powers, photomultiplier gain and detection filter settings. Images were analyzed using custom routines written in MATLAB. Briefly, images were first adjusted for brightness and contrast. A median filter was applied to the image to remove salt-and-pepper noise followed by Sobel filtering to determine the edges of the cells. This was followed by the following morphological operations: one round of dilation (imdilate function), one round of filling (imfill function) and two rounds of erosion (imerosion function). The cell positions were calculated as the centroids (regionprops function). The striatum was delineated and the number of centroids inside it was counted and divided by the total area of the region to give the cell density in cells per $\mathrm{mm}^{2}$. This analysis was repeated for all sections where the striatum was present and individual values for the sections were averaged to give a single value per animal.

Statistical analysis. All statistical analyses were performed using MATLAB (Supplementary Table 1). Group data are presented as boxplots. On each box, the central mark indicates the median and the top and bottom edges of the box indicate the 25th and 75th percentiles 
respectively. The whiskers extend to the data points not considered as outliers. Outliers were considered as points lying at $1.5 \mathrm{x} \mathrm{IQR}$ (inter-quartile range) above the 75th percentile or under the 25th percentile, thereby giving coverage of $\sim 99 \%$. The Anderson-Darling normality test was used to compare the empirical distribution of the data sets with a normal probability distribution. To compare normally distributed data, we used paired or unpaired two-tailed Student's t-test. To compare non-normally distributed data, we used Wilcoxon's rank-sum test for unpaired comparisons or Wilcoxon's signed-rank test for paired comparisons respectively. To analyze the differences among multiple experimental groups, we used a one-way analysis of variance (ANOVA) followed by the Tukey-Kramer post-hoc test. Statistical significance was considered at $\mathrm{p}$-values $\leq 0.05$. The number of animals for each experiment is described in each figure legend.

\section{References}

Favuzzi, E., Deogracias, R., Marques-Smith, A., Maeso, P., Jezequel, J., Exposito-Alonso, D., Balia, M., Kroon, T., Hinojosa, A.J., E, F.M., and Rico, B. (2019). Distinct molecular programs regulate synapse specificity in cortical inhibitory circuits. Science 363, 413-417.

Fremeau, R.T., Jr., Kam, K., Qureshi, T., Johnson, J., Copenhagen, D.R., Storm-Mathisen, J., Chaudhry, F.A., Nicoll, R.A., and Edwards, R.H. (2004). Vesicular glutamate transporters 1 and 2 target to functionally distinct synaptic release sites. Science 304, 1815-1819.

Gerfen, C.R., Paletzki, R., and Heintz, N. (2013). GENSAT BAC cre-recombinase driver lines to study the functional organization of cerebral cortical and basal ganglia circuits. Neuron 80, 1368-1383.

Goebbels, S., Bormuth, I., Bode, U., Hermanson, O., Schwab, M.H., and Nave, K.A. (2006). Genetic targeting of principal neurons in neocortex and hippocampus of NEX-Cre mice. Genesis 44, 611-621.

Heeroma, J.H., Roelandse, M., Wierda, K., van Aerde, K.I., Toonen, R.F., Hensbroek, R.A., Brussaard, A., Matus, A., and Verhage, M. (2004). Trophic support delays but does not prevent cell-intrinsic degeneration of neurons deficient for munc18-1. Eur. J. Neurosci. 20, 623-634.

Hnasko, T.S., Chuhma, N., Zhang, H., Goh, G.Y., Sulzer, D., Palmiter, R.D., Rayport, S., and Edwards, R.H. (2010). Vesicular glutamate transport promotes dopamine storage and glutamate corelease in vivo. Neuron 65, 643-656. 
Madisen, L., Zwingman, T.A., Sunkin, S.M., Oh, S.W., Zariwala, H.A., Gu, H., Ng, L.L., Palmiter, R.D., Hawrylycz, M.J., Jones, A.R., et al. (2010). A robust and high-throughput Cre reporting and characterization system for the whole mouse brain. Nat. Neurosci. 13, 133-140. Roth, B.L. (2016). DREADDs for Neuroscientists. Neuron 89, 683-694.

Takeuchi, O., Fisher, J., Suh, H., Harada, H., Malynn, B.A., and Korsmeyer, S.J. (2005).

Essential role of BAX,BAK in B cell homeostasis and prevention of autoimmune disease. Proc. Natl. Acad. Sci. USA 102, 11272-11277.

West, M.J., and Gundersen, H.J. (1990). Unbiased stereological estimation of the number of neurons in the human hippocampus. J. Comp. Neurol. 296, 1-22.

Xu, Q., Tam, M., and Anderson, S.A. (2008). Fate mapping Nkx2.1-lineage cells in the mouse telencephalon. J. Comp. Neurol. 506, 16-29.

Zolotukhin, S., Byrne, B.J., Mason, E., Zolotukhin, I., Potter, M., Chesnut, K., Summerford, C., Samulski, R.J., and Muzyczka, N. (1999). Recombinant adeno-associated virus purification using novel methods improves infectious titer and yield. Gene Ther 6, 973-985. 